\title{
Exchange option pricing under stochastic volatility: a correlation expansion
}

\author{
F. Antonelli · A. Ramponi · S. Scarlatti
}

Published online: 18 August 2009

(C) Springer Science+Business Media, LLC 2009

\begin{abstract}
Efficient valuation of exchange options with random volatilities while challenging at analytical level, has strong practical implications: in this paper we present a new approach to the problem which allows for extensions of previous known results. We undertake a route based on a multi-asset generalization of a methodology developed in Antonelli and Scarlatti (Finan Stoch 13:269-303, 2009) to handle simple European one-asset derivatives with volatility paths described by Ito's diffusive equations. Our method seems to adapt rather smoothly to the evaluation of Exchange options involving correlations among all the financial quantities that specify the model and it is based on expanding and approximating the theoretical evaluation formula with respect to correlation parameters. It applies to a whole range of models and does not require any particular distributional property. In order to test the quality of our approximation numerical simulations are provided in the last part of the paper.
\end{abstract}

Keywords Options $\cdot$ Stochastic volatility $\cdot$ SDE's $\cdot$ PDE's $\cdot$ Margrabe's formula

Mathematical Subject Classification (2002) $60 \mathrm{H} 10 \cdot 91 \mathrm{~B} 24$

JEL Classification (2007) C020 - G130

\section{Introduction}

It is well known among practitioners that, when the volatility of the underlying assets is allowed to vary stochastically, fast and accurate pricing of exotic products such as

F. Antonelli

Università di L'Aquila, L'Aquila, Italy

A. Ramponi · S. Scarlatti $(\bowtie)$

Università di Roma Tor-Vergata, Rome, Italy

e-mail: sergio.scarlatti@uniroma2.it 
barrier, Asian, basket or spread options must be solved, as a general rule, by applying the smartest Monte Carlo methodology at hand (we refer the reader to Glasserman 2004 for many possible implementations), since, in the quantitative finance literature, extensions from the Black \& Scholes setting to the stochastic volatility framework leading to closed or semi-closed pricing formulas are rare for plain vanilla claims and tend to disappear for more exotic derivatives.

In this paper we tackle the pricing of an exchange or Fisher-Margrabe option (Margrabe 1978; Fisher 1978), under stochastic volatility using an analytical approximation recently introduced in a work by Antonelli and Scarlatti (2009) to evaluate plain vanilla European call options, by writing a power series expansion of the no arbitrage price with respect to the correlation parameter between asset and volatility. It resulted fairly general, working for a variety of models, including the Stein and Stein (1991), the Heston (1993) and the Hull and White (1987) ones.

To the best of our knowledge, there are only few analytical results appearing in the financial literature on the pricing of exchange options, or closely related options, under stochastic volatility. The work of Duffie et al. (2000) where the price of a chooser option is obtained by transform methods in an affine setting. The paper by Bakshi and Madan (2000, Sect. 4) where, under the Heston dynamics, correlation options are discussed and the corresponding pricing problem is solved. We notice that correlation options can be related to exchange options, see e.g. Chung and Wang (2008) for an application to currency cross-rates. Our method is different: it suggests a first order approximation formula by expanding the option price with respect to all three correlation parameters $(\rho, \beta, v)$, representing, respectively, the asset to asset correlation and the correlations between the assets and their corresponding volatilities.

In Antonelli and Scarlatti (2009) the setting was one dimensional, but the ease of the method made us try an application to a multidimensional situation, such as the present one. The method is based on an iterative procedure. To identify the four coefficients characterizing the first order approximation formula, we first formally differentiate the associated backward Kolmogorov partial differential equation (PDE) satisfied by the price function and we specialize it at the zero values of the parameters. This gives birth to a series of chained PDE problems, whose solutions can be represented by means of the Feynman-Kaĉ formula, but they are difficult to be solved explicitly. Nevertheless, one may compute in approximated way those solutions step by step up to any desired order.

Luckily, as in the European case, the zeroth and first order terms are sufficient to obtain a good approximation of the price. This is probably due to the smallness of the parameters, so that the higher order terms usually add an error comparable with the computational one. Once identified these coefficients, to make them fully computable, we apply a further approximation by substituting the time average of future volatility with its mean as already suggested in the seminal paper by Hull and White (1987) and reconsidered by Alos (2006). To test the quality of the approximation we run numerical simulations in the case of the Hull and White model and in the case of the Heston model. In both cases we take the Monte Carlo simulations as our benchmarks. As expected, the method works naturally very well for small values of the correlations, but it results quite satisfactory also for real market values of $(\beta, v)$, usually quite large. Thus fixing those correlations on the market values, we provide tables for values of $\rho$ 
ranging from -0.5 to 0.5 , where we show that the relative error ranges from 0.001 to $8.57 \%$ for the Hull and White model and from 0.01 to $8.42 \%$ in the case of the Heston model, with only two exceptions.

The paper is organized as follows. In the next section we introduce general stochastic volatility model for the exchange option, the following one is devoted to the presentation of the method and the last to the numerical implementations.

\section{Stochastic volatility models for the exchange option}

In a market with two assets, an exchange option gives the holder the right, but not the obligation, to exchange at a future time $T$ one share of the underlying $S^{2}$ for one share of the underlying $S^{1}$. In the classical Black \& Scholes model, where the assets prices dynamics, with respect to a filtered probability space $\left(\Omega, \mathcal{F}, Q,\left\{\mathcal{F}_{t}\right\}_{t \geq 0}\right)$ are described by the equations

$$
d S_{t}^{1}=S_{t}^{1}\left(\mu_{1} d t+\sigma^{1} d W_{t}^{1}\right) \quad d S_{t}^{2}=S_{t}^{2}\left(\mu_{2} d t+\sigma^{2} d W_{t}^{2}\right)
$$

with $\left(W_{t}^{1}, W_{t}^{2}\right) \rho$-correlated Brownian motions, an explicit pricing formula for an exchange option was obtained by Margrabe (1978):

$$
u\left(t, \frac{S_{t}^{1}}{S_{t}^{2}}\right)=S_{t}^{2}\left[\frac{S_{t}^{1}}{S_{t}^{2}} \mathcal{N}\left(d_{1}\right)-\mathcal{N}\left(d_{2}\right)\right],
$$

where

$$
d_{1,2}=\frac{\log S_{t}^{1} / S_{t}^{2} \pm \Sigma(T-t) / 2}{\sqrt{\Sigma(T-t)}}, \quad \Sigma=\sigma_{1}^{2}-2 \rho \sigma_{1} \sigma_{2}+\sigma_{2}^{2} .
$$

It strongly resembles the classical Black \& Scholes formula and the correlation between assets gets incorporated in the volatility $\sqrt{\Sigma}$, see Poulsen (2009) for a recent survey on the topic.

We wish to extend the considerations that lead to Margrabe's formula (1) to a stochastic volatility setting, where the volatilities are expressed by means of Ito's diffusive equations. Since usually volatilities cannot be traded, we are naturally in conditions of incompleteness, but we maintain the no arbitrage assumption: we assume that a no arbitrage probability $P$, equivalent to $Q$ on $(\Omega, \mathcal{F})$, can be selected by some criterion so that our market model is described by the following SDE's

$$
\begin{array}{cl}
d S_{t}^{1}=S_{t}^{1}\left(r d t+f_{1}\left(v_{t}^{1}\right) d W_{t}^{1}\right), & d v_{t}^{1}=\eta_{1}\left(v_{t}^{1}\right) d t+\gamma_{1}\left(v_{t}^{1}\right) d B_{t}^{1} \\
d S_{t}^{2}=S_{t}^{2}\left(r d t+f_{2}\left(v_{t}^{2}\right) d Z_{t}\right), & d v_{t}^{2}=\eta_{2}\left(v_{t}^{2}\right) d t+\gamma_{2}\left(v_{t}^{2}\right) d B_{t}^{2}
\end{array}
$$

where $\left(W^{1}, Z, B^{1}, B^{2}\right)$ are correlated Brownian motions, $r>0$ is the risk-free rate and with initial conditions $S_{t}^{1}=s_{1}>0, S_{t}^{2}=s_{2}>0, v_{t}^{1}=y>0, v_{t}^{2}=z>0$.

We assume that the functions $f_{i}, \gamma_{i}, \eta_{i}: \mathbb{R} \longrightarrow \mathbb{R}$ are well defined and in $\mathcal{C}^{\infty}(\mathbb{R})$ with uniformly bounded derivatives of order greater than or equal 1 and that equations (2) and (3) have a unique strong solution $\left(S^{i}, v^{i}\right)$ that verifies for any $t \in[0, T]$ 


$$
E\left(\sup _{u \in[t, T]}\left(\left|S_{u}^{i}\right|^{2}+\left|v_{u}^{i}\right|^{2}\right)\right)<C
$$

for some constant $C$ depending on $T, s_{1}, s_{2}, y, z$.

The correlation structure may be modelled by four dimensional standard Brownian motion $\left(W^{1}, W^{2}, W^{3}, W^{4}\right)$ and 3 real parameters $-1<\rho, \beta, v<1$, by rewriting

$$
\begin{aligned}
& Z_{t}=\rho W_{t}^{1}+\sqrt{1-\rho^{2}} W_{t}^{2}, \quad B_{t}^{1}=v W_{t}^{1}+\sqrt{1-v^{2}} W_{t}^{3} \\
& B_{t}^{2}=\beta \rho W_{t}^{1}+\beta \sqrt{1-\rho^{2}} W_{t}^{2}+\sqrt{1-\beta^{2}} W_{t}^{4} .
\end{aligned}
$$

Remark 1 We would like to comment the foregoing hypothesis. When the coefficients $f_{i}$ are also bounded, the integrability requirement (4) is automatically satisfied by the solution (see Nualart (1996), Corollary 2.2.1). We do not want to introduce such an assumption as it would limit the models we may consider (see for example the Hull and White model). Under this generality, in some cases (4) does not follow, therefore we decided to include it as part of our running hypotheses.

Instead, some smoothness of the coefficients will be needed in several points to apply our method. For the sake of exposition we opted for $\mathcal{C}^{\infty}(\mathbb{R})$ regularity to be always able to take as many derivatives as desired.

Remark 2 We also would like to comment Eq. (5) which describe our correlation structure. First, it follows from (5) that $: \operatorname{cov}\left(W_{t}^{1}, Z_{t}\right)=\rho, \operatorname{cov}\left(W_{t}^{1}, B_{t}^{1}\right)=v$ and $\operatorname{cov}\left(Z_{t}, B_{t}^{2}\right)=\beta$. Second, in our model, the value of the correlation of the assets volatilities is implied by the previous equations and equal to $\rho \nu \beta$. We point out that our approach can handle correlation structures more general than the one discussed in this paper: for example, a 4-dimensional parametric description could also be studied exactly along the same lines as our 3-dimensional one. Finally we remark that our model with three correlation parameters closely resembles the two-assets log-normal model with stochastic volatility ${ }^{1}$ examinated by Bakshi and Madan (2000, Sect. 4) in their analysis of correlation options. There, the authors focus their study on the Heston model and solve the problem of determining in closed form the joint characteristic function which leads to explicit pricing formulas. Our stochastic equations $(2,3)$ describe a broader family of possible dynamics (including the Heston's one) depending on the choice of the functions $f_{i}, \eta_{i}, \gamma_{i}, i=1,2$. We then develop a unifying approach based on the idea of correlation expansion: partially because of that only approximate solutions to the pricing problem can be in general achieved. We notice that correlation options can be reconsidered under volatility dynamics different from that of Heston and then studied by the same methodology presented here: for these cases we do not expect to obtain solutions in closed or semi-closed form but computable first order approximations, in the correlation parameters, of the corresponding prices.

\footnotetext{
1 The dynamics considered in (Bakshi and Madan (2000)) can be obtained by collapsing the two SDE's for the volatilities appearing in (2) and (3) into a single equation and allowing, at the same time, for general correlations.
} 
Theorem 3 Let $[0, T]$ be a finite time interval. Under the previous hypotheses, the price of an exchange option with maturity $T$ in a market model defined by (2) and (3) is given by $s_{2} u\left(t, \frac{s_{1}}{s_{2}}, y, z ; \rho, \beta, v\right)$, where $u$ verifies the following Partial Differential Equation, provided it has a unique classical solution for $t \in[0, T], x \in \mathbb{R}, y, z>0$

$$
\left\{\begin{array}{l}
\frac{\partial u}{\partial t}+\frac{1}{2}\left[\left(f_{1}^{2}(y)-2 \rho f_{1}(y) f_{2}(z)+f_{2}^{2}(z)\right) \frac{\partial^{2} u}{\partial x^{2}}+\gamma_{1}^{2}(y) \frac{\partial^{2} u}{\partial y^{2}}+\gamma_{2}^{2}(z) \frac{\partial^{2} u}{\partial z^{2}}\right] \\
+v \gamma_{1}(y)\left[f_{1}(y)-\rho f_{2}(z)\right] \frac{\partial^{2} u}{\partial x \partial y}-\beta \gamma_{2}(z)\left[f_{2}(z)-\rho f_{1}(y)\right] \frac{\partial^{2} u}{\partial x \partial z}+\beta \rho v \gamma_{1}(y) \gamma_{2}(z) \frac{\partial^{2} u}{\partial y \partial z} \\
-\frac{1}{2}\left[f_{1}^{2}(y)-2 \rho f_{1}(y) f_{2}(z)+f_{2}^{2}(z)\right] \frac{\partial u}{\partial x}+\left[\eta_{1}(y)+v \rho \gamma_{1}(y) f_{2}(z)\right] \frac{\partial u}{\partial y} \\
+\left[\eta_{2}(z)+\beta \gamma_{2}(z) f_{2}(z)\right] \frac{\partial u}{\partial z}=0 \\
u(T, x, y, z ; \rho, \beta, v)=\left(\mathrm{e}^{x}-1\right)_{+} .
\end{array}\right.
$$

Proof A European exchange option with maturity $T$ has pay-off defined by

$$
\psi\left(S_{T}^{1}, S_{T}^{2}\right)=\left(S_{T}^{1}-S_{T}^{2}\right)^{+}=S_{T}^{2}\left(\frac{S_{T}^{1}}{S_{T}^{2}}-1\right)^{+}
$$

To price it, we may use the no arbitrage pricing principle. Indeed, by the Markovian nature of our system $(2,3)$, introducing the notation $\mathbf{E}_{\theta}$ for the expectation given the initial conditions at time $t, \theta=\left(s_{1}, s_{2}, y, z\right)$, the price of an exchange option at time $t$ may be written as

$$
\begin{aligned}
U\left(t, s_{1}, s_{2}, y, z ; \rho, \beta, v\right) & =\mathbf{E}_{\theta}\left(\mathrm{e}^{-r(T-t)} S_{T}^{2}\left(\frac{S_{T}^{1}}{S_{T}^{2}}-1\right)^{+}\right) \\
& =\mathbf{E}_{\theta}\left(\widetilde{S}_{T}^{2}\left(\frac{\widetilde{S}_{T}^{1}}{\widetilde{S}_{T}^{2}-1}\right)^{+}\right),
\end{aligned}
$$

where $\widetilde{S}_{T}^{i}=\mathrm{e}^{-r(T-t)} S_{T}^{i}, i=1,2$.

If we apply the Change-of-Numeraire technique, defining the probability measure $P^{*}$ equivalent to $P$ by

$\frac{d P^{*}}{d P}=\frac{\widetilde{S}_{T}^{2}}{s_{2}}=\exp \left\{-\frac{1}{2} \int_{t}^{T} f_{2}^{2}\left(v_{s}^{2}\right) d s+\int_{t}^{T} f_{2}\left(v_{s}^{2}\right)\left(\rho d W_{s}^{1}+\sqrt{1-\rho^{2}} d W_{s}^{2}\right)\right\}$,

we may rewrite (7) as

$$
\mathbf{E}_{\theta}\left(\widetilde{S}_{T}^{2}\left(\frac{\widetilde{S}_{T}^{1}}{\widetilde{S}_{T}^{2}-1}\right)^{+}\right)=s_{2} \mathbf{E}_{\theta}^{*}\left(\left(\frac{\widetilde{S}_{T}^{1}}{\widetilde{S}_{T}^{2}-1}\right)^{+}\right)
$$

where $E_{\theta}^{*}$ denotes the expectation with respect to $P^{*}$. From now on we shall omit the dependence on the initial data in the expectation. We remark that the assumed integrability of $S^{2}$ makes sure that the discounted process is a $P$-martingale (not only 
a local martingale) and that the Novikov condition, necessary to apply the change of probability, is verified. Besides, Girsanov theorem implies that the processes

$$
\bar{W}_{t}^{1}=W_{t}^{1}-\rho \int_{0}^{t} f_{2}\left(v_{s}^{2}\right) d s, \quad \bar{W}_{t}^{2}=W_{t}^{2}-\sqrt{1-\rho^{2}} \int_{0}^{t} f_{2}\left(v_{s}^{2}\right) d s
$$

are $P^{*}$-Brownian motions, which are also independent (indeed $<\bar{W}^{1}, \bar{W}^{2}>$. $\equiv 0$ ).

Introducing the process $X=\log \left(S^{1} / S^{2}\right)$, under $P^{*}$, the triple $\left(X, v^{1}, v^{2}\right)$ verifies the following equations

$$
\begin{aligned}
d X_{t}= & {\left[f_{1}\left(v_{t}^{1}\right)-\rho f_{2}\left(v_{t}^{2}\right)\right] d \bar{W}_{t}^{1}-\left(1-\rho^{2}\right)^{\frac{1}{2}} f_{2}\left(v_{t}^{2}\right) d \bar{W}_{t}^{2} } \\
& -\frac{1}{2}\left[f_{1}^{2}\left(v_{t}^{1}\right)-2 \rho f_{1}\left(v_{t}^{1}\right) f_{2}\left(v_{t}^{2}\right)+f_{2}^{2}\left(v_{t}^{2}\right)\right] d t \\
d v_{t}^{1}= & {\left[\eta_{1}\left(v_{t}^{1}\right)+v \rho \gamma_{1}\left(v_{t}^{1}\right) f_{2}\left(v_{t}^{2}\right)\right] d t+\gamma_{1}\left(v_{t}^{1}\right)\left(v d \bar{W}_{t}^{1}+\sqrt{1-v^{2}} d W_{t}^{3}\right) } \\
d v_{t}^{2}= & {\left[\eta_{2}\left(v_{t}^{2}\right)+\beta \gamma_{2}\left(v_{t}^{2}\right) f_{2}\left(v_{t}^{2}\right)\right] d t } \\
& +\gamma_{2}\left(v_{t}^{2}\right)\left(\beta \rho d \bar{W}_{t}^{1}+\beta \sqrt{1-\rho^{2}} d \bar{W}_{t}^{2}+\sqrt{1-\beta^{2}} d W_{t}^{4}\right) .
\end{aligned}
$$

Since (8), (9) and (10) form a Markovian system, we may use the flow notation for $X$ to express the dependence of the expectation upon the initial conditions $t, x=\frac{s_{1}}{s_{2}}$. Consequently the value of the option may be represented as $U\left(t, s_{1}, s_{2}, y, z ; \rho, \beta, v\right)=$ $s_{2} \mathbf{E}^{*}\left(\left(\mathrm{e}^{X_{T}^{t, x, y, z}}-1\right)^{+}\right)$. Focussing on the function

$$
u(t, x, y, z ; \rho, \beta, v):=\mathbf{E}^{*}\left(\left(\mathrm{e}^{X_{T}^{t, x, y, z}}-1\right)^{+}\right)
$$

due to our hypotheses, we know it is smooth in $(t, x, y, z)$ and, applying Itô's formula, we conclude it has to satisfy the evaluation $\operatorname{PDE}(6)$ for $t \in[0, T], x \in \mathbb{R}, y$, $z>0$.

The problem is now reduced to finding a solution, possibly an approximation of it, of the above PDE. In the next section we show how to produce a first order approximation that gives satisfactory numerical results.

\section{The correlation expansion approach}

In this section we mean to write an approximation for $u$, defined by (11), without using Fourier transform techniques. Since it can be shown that $u$ exhibits a smooth dependence on the correlation parameters, one may think to approximate it by its Taylor polynomial, of appropriate degree, in $\rho, \beta$, v written around some fixed initial values 
of them. Classically this expansion is considered around $(0,0,0)$, which corresponds to the no correlation situation, often easier to deal with.

Here, for exposition's sake, we construct only a first order approximation, which is often numerically satisfactory due to the smallness of the correlation parameters, even though theoretically the method may be pushed up to higher orders. Therefore, we propose the following approximation

$$
\begin{aligned}
u(t, x, y, z ; \rho, v, \beta) \approx & u(t, x, y, z ; 0,0,0)+\rho \frac{\partial u}{\partial \rho}(t, x, y, z ; 0,0,0) \\
& +v \frac{\partial u}{\partial v}(t, x, y, z ; 0,0,0)+\beta \frac{\partial u}{\partial \beta}(t, x, y, z ; 0,0,0) .
\end{aligned}
$$

and we want to identify the four coefficients of the polynomial. To do so, we are going to apply the method developed in Antonelli and Scarlatti (2009) in this multidimensional context.

Let us remark that, if we denote by $\mathcal{F}_{t, s}^{v^{1}, v^{2}}:=\sigma\left(v_{u}^{1}, v_{u}^{2}, t \leq u \leq s\right)$, then from (8) we have

$$
X_{s}^{t, x, y, z} \mid \mathcal{F}_{t, s}^{v^{1}, v^{2}} \sim \mathcal{N}\left(x-\frac{1}{2} \Sigma_{s}^{t, y, z}(\rho, v, \beta) ; \Sigma_{s}^{t, y, z}(\rho, v, \beta)\right)
$$

where

$$
\Sigma_{s}^{t, y, z}(\rho, v, \beta)=\int_{t}^{s}\left[f_{1}^{2}\left(v_{u}^{1, t, y}\right)-2 \rho f_{1}\left(v_{u}^{1, t, y}\right) f_{2}\left(v_{u}^{2, t, z}\right)+f_{2}^{2}\left(v_{u}^{2, t, z}\right)\right] d u
$$

So we are conditionally in the Black and Scholes framework and setting

$$
d_{1}(x, \Lambda)=\frac{x+\frac{1}{2} \Lambda}{\sqrt{\Lambda}}, \quad d_{2}(x, \Lambda)=\frac{x-\frac{1}{2} \Lambda}{\sqrt{\Lambda}}, \quad \Lambda>0
$$

we have that

$$
\begin{aligned}
u(t, x, y, z ; \rho, v, \beta)= & \mathbf{E}^{*}\left(\mathbf{E}^{*}\left(\left(\mathrm{e}^{X_{T}^{t, x, y, z}}-1\right)^{+} \mid \mathcal{F}_{t}^{v^{1}, v^{2}}\right)\right) \\
= & \mathrm{e}^{x} \mathbf{E}^{*}\left(\mathcal{N}\left(d_{1}\left(x, \Sigma_{T}^{t, y, z}(\rho, v, \beta)\right)\right)\right) \\
& -\mathbf{E}^{*}\left(\mathcal{N}\left(d_{2}\left(x, \Sigma_{T}^{t, y, z}(\rho, v, \beta)\right)\right)\right) .
\end{aligned}
$$

As said before, $u$ is solution of (6), which we rewrite in the following way

$$
\left\{\begin{array}{l}
\mathcal{L}_{0} u-\rho \mathcal{A} u+v \mathcal{B} u-\beta \mathcal{G} u-\rho v \mathcal{A}_{1} u+\rho \beta \mathcal{A}_{2} u+\rho v \beta \mathcal{A}_{3} u=0 \\
u(T, x, y, z ; \rho, v, \beta)=\left(\mathrm{e}^{x}-1\right)^{+}
\end{array}\right.
$$


where

$$
\begin{aligned}
& \mathcal{L}_{0}=\frac{\partial}{\partial t}+\frac{1}{2}\left[\left(f_{1}^{2}+f_{2}^{2}\right) \frac{\partial^{2}}{\partial x^{2}}+\gamma_{1}^{2} \frac{\partial^{2}}{\partial y^{2}}+\gamma_{2}^{2} \frac{\partial^{2}}{\partial z^{2}}\right]-\frac{f_{1}^{2}+f_{2}^{2}}{2} \frac{\partial}{\partial x}+\eta_{1} \frac{\partial}{\partial y}+\eta_{2} \frac{\partial}{\partial z} \\
& \mathcal{A}=f_{1} f_{2}\left(\frac{\partial^{2}}{\partial x^{2}}-\frac{\partial}{\partial x}\right), \quad \mathcal{B}=\gamma_{1} f_{1} \frac{\partial^{2}}{\partial x \partial y}, \quad \mathcal{G}=\gamma_{2} f_{2}\left(\frac{\partial^{2}}{\partial x \partial z}-\frac{\partial}{\partial z}\right) \\
& \mathcal{A}_{1}=\gamma_{1} f_{2}\left(\frac{\partial^{2}}{\partial x \partial y}-\frac{\partial}{\partial y}\right), \quad \mathcal{A}_{2}=\gamma_{2} f_{1} \frac{\partial^{2}}{\partial x \partial z}, \quad \mathcal{A}_{3}=\gamma_{1} \gamma_{2} \frac{\partial^{2}}{\partial y \partial z}
\end{aligned}
$$

By differentiating formally (13) and specializing the equations for $(\rho, \nu, \beta)=(0,0,0)$, one gets to the conclusion that

$$
\begin{aligned}
& u_{0}(t, x, y, z):=u(t, x, y, z ; 0,0,0), \quad u_{1}(t, x, y, z)=\frac{\partial u}{\partial \rho}(t, x, y, z ; 0,0,0), \\
& \phi_{1}(t, x, y, z):=\frac{\partial u}{\partial v}(t, x, y, z ; 0,0,0), \quad \psi_{1}(t, x, y, z):=\frac{\partial u}{\partial \beta}(t, x, y, z ; 0,0,0)
\end{aligned}
$$

solve, respectively, the following four PDE problems for $x \in \mathbb{R}, y, z>0$ and $t \in$ $[0, T]$

$$
\begin{aligned}
& \left\{\begin{array}{l}
\mathcal{L}_{0} u_{0}(t, x, y, z)=0 \\
u_{0}(T, x, y, z)=\left(\mathrm{e}^{x}-1\right)^{+}
\end{array}\right. \\
& \left\{\begin{array} { l } 
{ \mathcal { L } _ { 0 } \phi _ { 1 } ( t , x , y , z ) = - \mathcal { B } u _ { 0 } ( t , x , y , z ) } \\
{ \phi _ { 1 } ( T , x , y , z ) = 0 }
\end{array} \quad \left\{\begin{array}{l}
\mathcal{L}_{0} u_{1}(t, x, y, z)=\mathcal{A} u_{0}(t, x, y, z) \\
u_{1}(T, x, y, z)=0 .
\end{array}\right.\right. \\
&
\end{aligned}
$$

From now on we restrict our computations to the systems (14), since the same technique applies straightforward to the other two systems.

Exploiting Duhamel's principle (see Evans (1998), Chap. 2, Sects. 2.3-2.4), we know that

$$
u_{1}(t, x, y, z)=-\int_{t}^{T} u_{1}^{(\alpha)}(t, s, y, z) d \alpha
$$

where, for $t \in[0, \alpha), x \in \mathbb{R}, y, z>0, u_{1}^{(\alpha)}$ is solution of

$$
\left\{\begin{array}{l}
\mathcal{L}_{0} u_{1}^{(\alpha)}(t, x, y, z)=0 \\
u_{1}^{(\alpha)}(\alpha, x, y, z)=\mathcal{A} u_{0}(\alpha, x, y, z)
\end{array}\right.
$$

Therefore we may apply the Feynmann-Kaĉ formula to obtain the probabilistic representation of the solution

$$
u_{1}^{(\alpha)}(t, x, y, z)=\mathbf{E}^{*}\left[\left(\mathcal{A} u_{0}\right)\left(\alpha, X_{\alpha}^{t, x, y, z}, v_{\alpha}^{1, t, y}, v_{\alpha}^{2, t, z}\right)\right]
$$


We remark that in the equation above the processes representing the state variables are to be intended at correlations $(0,0,0)$. We know that first term of the expansion is given by

$$
\begin{aligned}
u_{0}(t, x, y, z) & =u(t, x, y, z ; 0,0,0) \\
& =\mathbf{E}^{*}\left[\mathrm{e}^{x} \mathcal{N}\left(d_{1}\left(x, \Sigma_{T}^{t, y, z}(0,0,0)\right)\right)-\mathcal{N}\left(d_{2}\left(x, \Sigma_{T}^{t, y, z}(0,0,0)\right)\right)\right],
\end{aligned}
$$

(from now on we omit the argument $(0,0,0)$ in $\left.\Sigma_{T}^{t, y, z}\right)$ so computing the derivatives we have

$$
\begin{aligned}
\frac{\partial u_{0}}{\partial x}= & \mathrm{e}^{x} \mathbf{E}^{*}\left[\mathcal{N}\left(d_{1}\left(x, \Sigma_{T}^{t, y, z}\right)\right)\right], \frac{\partial^{2} u_{0}}{\partial x^{2}}=\mathrm{e}^{x}\left\{\mathbf { E } ^ { * } \left[\mathcal{N}\left(d_{1}\left(x, \Sigma_{T}^{t, y, z}\right)\right)\right.\right. \\
& \left.\left.+\mathcal{N}^{\prime}\left(d_{1}\left(x, \Sigma_{T}^{t, y, z}\right)\right) \frac{\partial d_{1}}{\partial x}\left(x, \Sigma_{T}^{t, y, z}\right)\right]\right\}
\end{aligned}
$$

which imply

$$
\frac{\partial^{2} u_{0}}{\partial x^{2}}-\frac{\partial u_{0}}{\partial x}=e^{x} \mathbf{E}^{*}\left[\mathcal{N}^{\prime}\left(d_{1}\left(x, \Sigma_{T}^{t, y, z}\right)\right) \frac{1}{\sqrt{\Sigma_{T}^{t, y, z}}}\right] .
$$

Substituting back in our formula (16), we obtain

$$
\begin{aligned}
u_{1}(t, x, y, z)= & -\int_{t}^{T} \mathbf{E}^{*}\left[\left(\mathcal{A} u_{0}\right)\left(\alpha, X_{\alpha}^{t, x, y, z}, v_{\alpha}^{1, t, y}, v_{\alpha}^{2, t, z}\right)\right] d \alpha \\
= & -\int_{t}^{T} \mathbf{E}^{*}\left[f_{1}\left(v_{\alpha}^{1, t, y}\right) f_{2}\left(v_{\alpha}^{2, t, z}\right) \mathrm{e}^{X_{\alpha}^{t, x, y, z}}\right. \\
& \times \mathbf{E}^{*}\left[\frac{\mathcal{N}^{\prime}\left(d_{1}\left(X_{\alpha}^{t, x, y, z}, \Sigma_{T}^{\alpha, v_{\alpha}^{1, t, y}, v_{\alpha}^{2, t, z}}\right)\right)}{\left.\left.\sqrt{\Sigma_{T}^{\alpha, v_{\alpha}^{1, t, y}, v_{\alpha}^{2, t, z}}}\right]\right] d \alpha .} .\right.
\end{aligned}
$$

But the internal expectation is actually conditioned w.r.t. $X_{\alpha}^{t, x, y, z}, v_{\alpha}^{1, t, y}, v_{\alpha}^{2, t, z}$, hence noticing that $\mathrm{e}^{x} \mathcal{N}^{\prime}\left(d_{1}\right)=\mathcal{N}^{\prime}\left(d_{2}\right)$, we may write 


$$
\begin{aligned}
& \mathbf{E}^{*}\left[f_{1}\left(v_{\alpha}^{1, t, y}\right) f_{2}\left(v_{\alpha}^{2, t, z}\right) \mathrm{e}^{X_{\alpha}^{t, x, y, z}}\right. \\
& \left.\times \mathbf{E}^{*}\left[\mathcal{N}^{\prime}\left(d_{1}\left(X_{\alpha}^{t, x, y, z}, \Sigma_{T}^{\alpha, v_{\alpha}^{1, t, y}, v_{\alpha}^{2, t, z}}\right)\right) \frac{1}{\sqrt{\Sigma_{T}^{\alpha, v_{\alpha}^{1, t, y}, v_{\alpha}^{2, t, z}}}}\right]\right] \\
& =\mathbf{E}^{*}\left[f_{1}\left(v_{\alpha}^{1, t, y}\right) f_{2}\left(v_{\alpha}^{2, t, z}\right) \mathrm{e}^{X_{\alpha}^{t, x, y, z}} \mathcal{N}^{\prime}\left(d_{1}\left(X_{\alpha}^{t, x, y, z}, \Sigma_{T}^{\alpha, v_{\alpha}^{1, t, y}, v_{\alpha}^{2, t, z}}\right)\right)\right. \\
& \left.\times \frac{1}{\sqrt{\Sigma_{T}^{\alpha, v_{\alpha}^{1, t, y}, v_{\alpha}^{2, t, z}}}}\right] \\
& =\mathbf{E}^{*}\left[f_{1}\left(v_{\alpha}^{1, t, y}\right) f_{2}\left(v_{\alpha}^{2, t, z}\right) \frac{1}{\sqrt{\Sigma_{T}^{\alpha, v_{\alpha}^{1, t, y}, v_{\alpha}^{2, t, z}}}}\right. \\
& \left.\times \mathbf{E}^{*}\left[\mathrm{e}^{X_{\alpha}^{t, x, y, z}} \mathcal{N}^{\prime}\left(d_{1}\left(X_{\alpha}^{t, x, y, z}, \Sigma_{T}^{\alpha, v_{\alpha}^{1, t, y}, v_{\alpha}^{2, t, z}}\right)\right) \mid \mathcal{F}_{T}^{v^{1}, v^{2}}\right]\right] \\
& =\mathbf{E}^{*}\left[f_{1}\left(v_{\alpha}^{1, t, y}\right) f_{2}\left(v_{\alpha}^{2, t, z}\right) \frac{1}{\sqrt{\Sigma_{T}^{\alpha, v_{\alpha}^{1, t, y}, v_{\alpha}^{2, t, z}}}}\right. \\
& \left.\times \mathbf{E}^{*}\left[\mathcal{N}^{\prime}\left(d_{2}\left(X_{\alpha}^{t, x, y, z}, \Sigma_{T}^{\alpha, v_{\alpha}^{1, t, y}, v_{\alpha}^{2, t, z}}\right)\right) \mid \mathcal{F}_{T}^{v^{1}, v^{2}}\right]\right] .
\end{aligned}
$$

Our processes are Markovian and the flow property implies that $\Sigma_{T}^{\alpha, v_{\alpha}^{1, t, y}, v_{\alpha}^{2, t, z}}=$ $\Sigma_{[\alpha, T]}^{t, y, z}$, where by the subindex $[\alpha, T]$ we mean the integral extended to such interval. Thus, knowing that $X_{\alpha}^{t, x, y, z} \mid \mathcal{F}_{T}^{v^{1}, v^{2}} \sim \mathcal{N}\left(x-\frac{1}{2} \Sigma_{\alpha}^{t, y, z} ; \Sigma_{\alpha}^{t, y, z}\right)$, it results

$$
d_{2}\left(X_{\alpha}^{t, x, y, z}, \Sigma_{[\alpha, T]}^{t, y, z}\right) \mid \mathcal{F}_{T}^{v^{1}, v^{2}} \sim \mathcal{N}\left(\frac{x-\frac{1}{2} \Sigma_{T}^{t, y, z}}{\sqrt{\Sigma_{[\alpha, T]}^{t, y, z}}} ; \frac{\Sigma_{\alpha}^{t, y, z}}{\Sigma_{[\alpha, T]}^{t, y, z}}\right) .
$$

Since $\mathcal{N}^{\prime}\left(d_{2}\right)=\exp \left(-d_{2}^{2} / 2\right) / \sqrt{2 \pi}$ and for a Gaussian r.v. $\Gamma \sim \mathcal{N}\left(\mu, \sigma^{2}\right)$ is

$$
\frac{1}{\sqrt{2 \pi}} \mathbf{E}\left[\mathrm{e}^{-\Gamma^{2} / 2}\right]=\frac{\mathrm{e}^{-\mu^{2} / 2\left(\sigma^{2}+1\right)}}{\sqrt{\left(\sigma^{2}+1\right)(2 \pi)}}
$$


we have

$\frac{1}{\sqrt{2 \pi}} \mathbf{E}^{*}\left[\exp \left(-\frac{1}{2} d_{2}^{2}\left(X_{\alpha}^{t, x, y, z}, \Sigma_{[\alpha, T]}^{t, y, z}\right)\right) \mid \mathcal{F}_{T}^{v^{1}, v^{2}}\right]=\frac{1}{\sqrt{2 \pi}} \frac{\sqrt{\Sigma_{[\alpha, T]}^{t, y, z}}}{\sqrt{\Sigma_{T}^{t, y, z}}} \mathrm{e}^{-\frac{d_{2}^{2}}{2}\left(t, x, \Sigma_{T}^{t, y, z}\right)}$

and finally we arrive at the expression

$$
u_{1}(t, x, y, z)=-\int_{t}^{T} \mathbf{E}^{*}\left[f_{1}\left(v_{\alpha}^{1, t, y}\right) f_{2}\left(v_{\alpha}^{2, t, z}\right) \frac{\mathcal{N}^{\prime}\left(d_{2}\left(x, \Sigma_{T}^{t, y, z}\right)\right)}{\sqrt{\Sigma_{T}^{t, y, z}}}\right] d \alpha
$$

Focussing now on the systems (15) and running calculations similar to those shown above, we arrive at the following expression for the first order terms with respect to $v$ and $\beta$

$$
\begin{aligned}
\phi_{1}(t, x, y, z)= & -\int_{t}^{T} \int_{\alpha}^{T} \mathbf{E}^{*}\left[\left(\gamma_{1} f_{1}\right)\left(v_{\alpha}^{1, t, y}\right)\left(f_{1} f_{1}^{\prime}\right)\left(v_{\alpha_{1}}^{1, t, y}\right) \frac{\partial v_{\left[\alpha, \alpha_{1}\right]}^{1, t, y}}{\partial y}\right. \\
& \left.\times \frac{\mathcal{N}^{\prime}\left(d_{2}\left(x, \Sigma_{T}^{t, y, z}\right)\right) d_{2}\left(x, \Sigma_{T}^{t, y, z}\right)}{\Sigma_{T}^{t, y, z}}\right] d \alpha_{1} d \alpha
\end{aligned}
$$

and

$$
\begin{aligned}
\psi_{1}(t, x, y, z)= & \int_{t}^{T} \int_{\alpha}^{T} \mathbf{E}^{*}\left[\left(\gamma_{2} f_{2}\right)\left(v_{\alpha}^{2, t, z}\right)\left(f_{2} f_{2}^{\prime}\right)\left(v_{\alpha_{1}}^{2, t, z}\right) \frac{\partial v_{\left[\alpha, \alpha_{1}\right]}^{2, t, z}}{\partial z}\right. \\
& \left.\times \frac{\mathcal{N}^{\prime}\left(d_{2}\left(x, \Sigma_{T}^{t, y, z}\right)\right) d_{1}\left(x, \Sigma_{T}^{t, y, z}\right)}{\Sigma_{T}^{t, y, z}}\right] d \alpha_{1} d \alpha
\end{aligned}
$$

where for $w_{1}=y, w_{2}=z$ we have

$$
\begin{aligned}
\frac{\partial v_{\left[\alpha, \alpha_{1}\right]}^{i, t, w_{i}}}{\partial w_{i}}=\exp & \left\{\int_{\alpha}^{\alpha_{1}}\left[\eta_{i}^{\prime}\left(v_{\alpha_{2}}^{i, t, w_{i}}\right)-\frac{1}{2}\left(\gamma_{i}^{\prime}\left(v_{\alpha_{2}}^{i, t, w_{i}}\right)\right)^{2}\right] d \alpha_{2}\right. \\
& \left.+\int_{\alpha}^{\alpha_{1}} \gamma_{i}^{\prime}\left(v_{\alpha_{2}}^{i, t, w_{i}}\right) d W_{\alpha_{2}}^{i+2}\right\} .
\end{aligned}
$$

As already mentioned, we point out that this method may be pushed further than the first order and in the appendix we show how to compute the second order derivatives. Nevertheless, the first order terms are expected weight the most, as the higher order 
terms of the expansion will be multiplied by powers greater than 2 of the correlation parameters, which, being naturally small, will probably reduce their numerical contributions.

We also remark that the successive derivatives in only one of the parameters can be computed recursively. For instance, differentiating repeatedly (13) in $\rho$ and specializing the result at $(0,0,0)$, it turns out that the coefficients

$$
u_{k}(t, x, y, z)=\frac{1}{k !} \frac{\partial^{k} u(t, x, y, z ; 0,0,0)}{\partial \rho^{k}},
$$

for $x \in \mathbb{R}, y, z>0$, are solutions of

$$
\left\{\begin{array} { l } 
{ \mathcal { L } _ { 0 } u _ { 0 } ( t , x , y , z ) = 0 } \\
{ u _ { 0 } ( T , x , y , z ) = ( \mathrm { e } ^ { x } - 1 ) ^ { + } }
\end{array} \quad \left\{\begin{array}{l}
\mathcal{L}_{0} u_{k}(t, x, y, z)=\mathcal{A} u_{k-1}(t, x, y, z) \\
u_{k}(T, x, y, z)=0
\end{array}\right.\right.
$$

Again Duhamel's principle and Feynmann-Kaĉ's representation give

$$
u_{k}^{(\alpha)}(t, x, y, z)=\int_{t}^{T} \mathbf{E}^{*}\left[\left(\mathcal{A} u_{k-1}\right)\left(\alpha, X_{\alpha}^{t, x, y, z}, v_{\alpha}^{1, t, y}, v_{\alpha}^{2, t, z}\right)\right] d \alpha
$$

providing a tool to compute the coefficients up to any order.

Remark 4 If two of the parameters are zero, say $v$ and $\beta$, introducing the further hypothesis $\left[\Sigma_{s}^{t, y, z}(\rho, 0,0)\right]^{-1} \in \bigcap_{p \geq 1} L^{p}$, then the convergence of the power series in the $\rho$ parameter

$$
u(t, x, y, z ; \rho, 0,0)=\sum_{k \geq 0} \frac{1}{k !} \frac{\partial^{k} u(t, x, y, z ; 0,0,0)}{\partial \rho^{k}} \rho^{k},
$$

is ensured within an appropriate radius of convergence (see Antonelli and Scarlatti (2009) for the details).

\section{The method in practice}

In this section we apply the method exposed so far to two popular models: Heston and Hull and White. Here, when needed, we index the initial conditions as $w_{1}=y$ and $w_{2}=z$.

First, we have to introduce a further approximation to make the coefficients totally computable, since the joint distributions necessary to compute $u_{0}, u_{1}, \phi_{1}$ and $\psi_{1}$ are usually unknown. This approximation is of the same type as the one appearing in Antonelli and Scarlatti (2009), which also contains an estimation of the relative error. 
If $\Sigma_{T}^{t, y, z}$ is a random variable sufficiently concentrated around its mean, one may think of approximating it by its expectation

$$
\Sigma_{T}^{t, y, z} \rightarrow \bar{\Sigma}_{T}^{t, y, z}=\mathbf{E}^{*}\left(\Sigma_{T}^{t, y, z}\right)=\int_{t}^{T}\left(\mathbf{E}^{*}\left(f_{1}^{2}\left(v_{s}^{1, t, y}\right)\right)+\mathbf{E}^{*}\left(f_{2}^{2}\left(v_{s}^{2, t, z}\right)\right)\right) d s,
$$

that leads to the following natural approximations

$$
\begin{aligned}
\bar{d}_{i}= & d_{i}\left(x, \bar{\Sigma}_{T}^{t, y, z}\right), \quad i=1,2 \\
\bar{u}_{0}(t, x, y, z)= & \left(\mathrm{e}^{x} \mathcal{N}\left(\bar{d}_{1}\right)-\mathcal{N}\left(\bar{d}_{2}\right)\right) \\
\bar{u}_{1}(t, x, y, z)= & -\frac{\mathcal{N}^{\prime}\left(\bar{d}_{2}\right)}{\sqrt{\bar{\Sigma}_{T}^{t, y, z}}} \int_{t}^{T} \mathbf{E}^{*}\left(f_{1}\left(v_{\alpha}^{1, t, y}\right) f_{2}\left(v_{\alpha}^{2, t, z}\right)\right) d \alpha, \\
\bar{\phi}_{1}(t, x, y, z)= & -\frac{\mathcal{N}^{\prime}\left(\bar{d}_{2}\right) \bar{d}_{2}}{\bar{\Sigma}_{T}^{t, y, z}} \int_{t}^{T} \int_{\alpha}^{T} \mathbf{E}^{*} \\
& \times\left[\left(\gamma_{1} f_{1}\right)\left(v_{\alpha}^{1, t, y}\right)\left(f_{1} f_{1}^{\prime}\right)\left(v_{\alpha_{1}}^{1, t, y}\right) \frac{\partial v_{\left[\alpha, \alpha_{1}\right]}^{1, t, y}}{\partial y}\right] d \alpha_{1} d \alpha, \\
\bar{\psi}_{1}(t, x, y, z)= & \frac{\mathcal{N}^{\prime}\left(\bar{d}_{2}\right) \bar{d}_{1}}{\bar{\Sigma}_{T}^{t, y, z}} \int_{t}^{T} \int_{\alpha}^{T} \mathbf{E}^{*} \\
& \times\left[\left(\gamma_{2} f_{2}\right)\left(v_{\alpha}^{2, t, z}\right)\left(f_{2} f_{2}^{\prime}\right)\left(v_{\alpha_{1}}^{2, t, z}\right) \frac{\partial v_{\left[\alpha, \alpha_{1}\right]}^{2, t, z}}{\partial z}\right] d \alpha_{1} d \alpha .
\end{aligned}
$$

which may be explicitly computed in the models mentioned above.

Of course, substituting a random variable with its mean is a very rough approximation. The biggest part of the error is carried by the 0th term which is not mitigated by any power of the correlation parameters. We therefore propose to refine the approximation of $u_{0}$, by pushing the Taylor expansion around the expected value of $\Sigma$ to the second order. Indeed, looking at $u_{0}$, one realizes that it is defined as

$$
u_{0}(t, x, y, z)=\mathbf{E}^{*}\left(F\left(\Sigma_{T}^{t, y, z}\right)\right)
$$

where $F(\sigma)=\mathrm{e}^{x} \mathcal{N}\left(d_{1}(x, \sigma)\right)-\mathcal{N}\left(d_{2}(x, \sigma)\right)$ is regular in $\sigma$ and it can be expanded up to any desired order. In particular

$$
F^{\prime}(\sigma)=\frac{1}{2 \sqrt{\sigma}} \mathcal{N}^{\prime}\left(d_{2}(x, \sigma)\right), \quad F^{\prime \prime}(\sigma)=\frac{1}{4 \sigma^{3 / 2}} \mathcal{N}^{\prime}\left(d_{2}\right)\left[d_{1} d_{2}-1\right](x, \sigma)
$$


Therefore approximating $F(\sigma)$ with its second order expansion around $\bar{\Sigma}_{T}^{t, y, z}$ and applying it to $\Sigma_{T}^{t, y, z}$, we obtain

$$
\begin{aligned}
\left.F\left(\Sigma_{T}^{t, y, z}\right)\right) \approx & F\left(\bar{\Sigma}_{T}^{t, y, z}\right)+F^{\prime}\left(\bar{\Sigma}_{T}^{t, y, z}\right)\left(\Sigma_{T}^{t, y, z}-\bar{\Sigma}_{T}^{t, y, z}\right) \\
& +\frac{1}{2} F^{\prime \prime}\left(\bar{\Sigma}_{T}^{t, y, z}\right)\left(\Sigma_{T}^{t, y, z}-\bar{\Sigma}_{T}^{t, y, z}\right)^{2},
\end{aligned}
$$

taking expectations the second term vanishes and we define

$$
\tilde{u}_{0}(t, x, y, z)=\bar{u}_{0}(t, x, y, z)+\frac{\mathcal{N}^{\prime}\left(\bar{d}_{2}\right)}{8\left(\bar{\Sigma}_{T}^{t, y, z}\right)^{3 / 2}}\left(\bar{d}_{2} \bar{d}_{1}-1\right) \operatorname{var}\left(\Sigma_{T}^{t, y, z}\right),
$$

leading to the following approximation formula

$$
\begin{aligned}
u(t, x, y, z ; \rho, v, \beta) \approx & \tilde{u}_{0}(t, x, y, z)+\rho \bar{u}_{1}(t, x, y, z)+v \bar{\phi}_{1}(t, x, y, z) \\
& +\beta \bar{\psi}_{1}(t, x, y, z) .
\end{aligned}
$$

In the following we present a concrete application of such a formula by using the well-known stochastic volatility models proposed by Hull and White (1987) and Heston (1993). The first one is a fully non-affine model for all values of the parameters $\rho, \beta, v$, while the second is affine only for $\rho=0$. Even if recently Maghsoodi (2007) presented an analytic solution to the pricing problem in the correlated Hull and White model based on a representation of the Hartman-Watson distribution, the eventual extension to the two-dimensional setting does not appear to be straightforward. Approximation techniques still remain a valuable alternative to Monte Carlo methods.

\subsection{Application to the Hull and White model}

The model is defined by the following choices

$$
f_{i}(v)=v ; \quad \eta_{i}(v)=\eta_{i} v ; \quad \gamma_{i}(v)=\gamma_{i} v, \quad \eta_{i}, \gamma_{i} \in \mathbb{R}, \quad i=1,2
$$

and Eqs. 2 and 3 become

$$
\begin{aligned}
& d S_{t}^{1}=S_{t}^{1}\left(r d t+v_{t}^{1} d W_{t}^{1}\right), \quad d v_{t}^{1}=v_{t}^{1}\left(\eta_{1} d t+\gamma_{1} d B_{t}^{1}\right) \\
& d S_{t}^{2}=S_{t}^{2}\left(r d t+v_{t}^{2} d Z_{t}\right), \quad d v_{t}^{2}=v_{t}^{2}\left(\eta_{2} d t+\gamma_{2} d B_{t}^{2}\right)
\end{aligned}
$$

The volatilities are therefore geometric Brownian motions. We remark that to guarantee the martingale property for the asset prices, it is necessary to have negative correlations between assets and volatilities (see Heyde and Wong 2004 for details). 
Looking at the uncorrelated case, needed to compute the coefficients, we have that the volatilities are

$$
v_{\alpha}^{1, t, y}=y \mathrm{e}^{\left(\eta_{1}-\frac{\gamma_{1}^{2}}{2}\right)(\alpha-t)+\gamma_{1}\left(W_{\alpha}^{3}-W_{t}^{3}\right)}, \quad v_{\alpha}^{2, t, z}=z \mathrm{e}^{\left(\eta_{2}-\frac{\gamma_{2}^{2}}{2}\right)(\alpha-t)+\gamma_{1}\left(W_{\alpha}^{4}-W_{t}^{4}\right)},
$$

hence $\mathbf{E}^{*}\left(\left(v_{\alpha}^{i, t, w_{i}}\right)^{p}\right)=w_{i}^{p} \mathrm{e}^{\left[p \eta_{i}+p(p-1) \frac{\gamma_{i}^{2}}{2}\right](\alpha-t)}$, for $i=1,2$ and any $p \geq 1$. This leads to the following approximations

$$
\bar{\Sigma}_{T}^{t, y, z}=y^{2} \frac{\mathrm{e}^{\left(2 \eta_{1}+\gamma_{1}^{2}\right)(T-t)}-1}{2 \eta_{1}+\gamma_{1}^{2}}+z^{2} \frac{\mathrm{e}^{\left(2 \eta_{2}+\gamma_{2}^{2}\right)(T-t)}-1}{2 \eta_{2}+\gamma_{2}^{2}}, \bar{d}_{h}=d_{h}\left(x, \bar{\Sigma}_{T}^{t, y, z}\right), \quad h=1,2
$$

which, substituted in (23-26), give

$$
\begin{aligned}
\bar{u}_{0}(t, x, y, z)= & \mathrm{e}^{x} \mathcal{N}\left(\bar{d}_{1}\right)-\mathcal{N}\left(\bar{d}_{2}\right), \quad \bar{u}_{1}(t, x, y, z)=-y z \frac{\mathcal{N}^{\prime}\left(\bar{d}_{2}\right)}{\bar{\Sigma}_{T}^{t, y, z}} \frac{\mathrm{e}^{\left(\eta_{1}+\eta_{2}\right)(T-t)}-1}{\eta_{1}+\eta_{2}}, \\
\bar{\phi}_{1}(t, x, y, z)= & -\frac{y^{3} \gamma_{1} \mathcal{N}^{\prime}\left(\bar{d}_{2}\right) \bar{d}_{2}}{\left(2 \eta_{1}+\gamma_{1}^{2}\right) \bar{\Sigma}_{T}^{t, y, z}} \\
& \times\left[\frac{\mathrm{e}^{\left(3 \eta_{1}+3 \gamma_{1}^{2}\right)(T-t)}\left(2 \eta_{1}+\gamma_{1}^{2}\right)}{\left(\eta_{1}+2 \gamma_{1}^{2}\right)\left(3 \eta_{1}+3 \gamma_{1}^{2}\right)}-\frac{\mathrm{e}^{\left(2 \eta_{1}+\gamma_{1}^{2}\right)(T-t)}}{\eta_{1}+2 \gamma_{1}^{2}}+\frac{1}{3 \eta_{1}+3 \gamma_{1}^{2}}\right] . \\
\bar{\psi}_{1}(t, x, y, z)= & \frac{z^{3} \gamma_{2} \mathcal{N}^{\prime}\left(\bar{d}_{2}\right) \bar{d}_{1}}{\left(2 \eta_{2}+\gamma_{2}^{2}\right) \bar{\Sigma}_{T}^{t, y, z}} \\
& \times\left[\frac{\mathrm{e}^{\left(3 \eta_{2}+3 \gamma_{2}^{2}\right)(T-t)}\left(2 \eta_{2}+\gamma_{2}^{2}\right)}{\left(\eta_{2}+2 \gamma_{2}^{2}\right)\left(3 \eta_{2}+3 \gamma_{2}^{2}\right)}-\frac{\mathrm{e}^{\left(2 \eta_{2}+\gamma_{2}^{2}\right)(T-t)}}{\eta_{2}+2 \gamma_{2}^{2}}+\frac{1}{3 \eta_{2}+3 \gamma_{2}^{2}}\right] .
\end{aligned}
$$

In this case we want to apply a better refinement of the zeroth term.

To compute the 1-order approximation (27) for $u_{0}$, we first need to evaluate the variance of $\Sigma_{T}^{t, y, z}$

$$
\operatorname{var}\left(\Sigma_{T}^{t, y, z}\right)=\mathbf{E}\left[\left(\Sigma_{T}^{t, y, z}\right)^{2}\right]-\left(\bar{\Sigma}_{T}^{t, y, z}\right)^{2}
$$


We only need to compute the first term

$$
\begin{aligned}
\mathbf{E}\left(\left(\Sigma_{T}^{t, y, z}\right)^{2}\right)= & \mathbf{E}\left(\int_{t}^{T}\left[\left(v_{s}^{1, t, y}\right)^{2}+\left(v_{s}^{2, t, z}\right)^{2}\right] d s \int_{t}^{T}\left[\left(v_{\alpha}^{1, t, y}\right)^{2}+\left(v_{\alpha}^{2, t, z}\right)^{2}\right] d \alpha\right) \\
= & \int_{t}^{T} \int_{t}^{T} \mathbf{E}\left[\left(v_{s}^{1, t, y} v_{\alpha}^{1, t, y}\right)^{2}\right] d s d \alpha+\int_{t}^{T} \int_{t}^{T} \mathbf{E}\left[\left(v_{s}^{2, t, z} v_{\alpha}^{2, t, z}\right)^{2}\right] d s d \alpha \\
& +2 \int_{t}^{T} \mathbf{E}\left[\left(v_{\alpha}^{1, t, y}\right)^{2}\right] d s \int_{t}^{T} \mathbf{E}\left[\left(v_{\alpha}^{2, t, z}\right)^{2}\right] d \alpha .
\end{aligned}
$$

For $i=1,2$, if $s>\alpha$ then $\mathbf{E}\left[\left(v_{s}^{i, t, w_{i}} v_{\alpha}^{i, t, w_{i}}\right)^{2}\right]=w_{i}^{4} \mathrm{e}^{\left(4 \eta_{i}+6 \gamma_{i}^{2}\right)(\alpha-t)+\left(2 \eta_{i}+\gamma_{i}^{2}\right)(s-\alpha)}$, otherwise if $s<\alpha \mathbf{E}\left[\left(v_{s}^{i, t, w_{i}} v_{\alpha}^{i, t, w_{i}}\right)^{2}\right]=w_{i}^{4} \mathrm{e}^{\left(4 \eta_{i}+6 \gamma_{i}^{2}\right)(s-t)+\left(2 \eta_{i}+\gamma_{i}^{2}\right)(\alpha-s)}$, both giving the same integral. Thus we may conclude

$$
\begin{aligned}
\mathbf{E}\left(\left(\Sigma_{T}^{t, y, z}\right)^{2}\right)=2 & \left\{\int _ { t } ^ { T } \int _ { t } ^ { s } \left[y^{4} \mathrm{e}^{\left(2 \eta_{1}+\gamma_{1}^{2}\right)(s-t)+\left(2 \eta_{1}+5 \gamma_{1}^{2}\right)(\alpha-t)}\right.\right. \\
& \left.+z^{4} \mathrm{e}^{\left(2 \eta_{2}+\gamma_{2}^{2}\right)(s-t)+\left(2 \eta_{2}+5 \gamma_{2}^{2}\right)(\alpha-t)}\right] d \alpha d s \\
& \left.+y^{2} z^{2} \int_{t}^{T} \mathrm{e}^{\left(2 \eta_{1}+\gamma_{1}^{2}\right)(s-t)} d s \int_{t} \mathrm{e}^{\left(2 \eta_{2}+\gamma_{2}^{2}\right)(\alpha-t)} d \alpha\right\} \\
=2\left\{y^{4}\left[\frac{\mathrm{e}^{\left(4 \eta_{1}+6 \gamma_{1}^{2}\right)(T-t)}-1}{\left(2 \eta_{1}+5 \gamma_{1}^{2}\right)\left(4 \eta_{1}+6 \gamma_{1}^{2}\right)}-\frac{\mathrm{e}^{\left(2 \eta_{1}+\gamma_{1}^{2}\right)(T-t)}-1}{\left(2 \eta_{1}+5 \gamma_{1}^{2}\right)\left(2 \eta_{1}+\gamma_{1}^{2}\right)}\right]\right. & \\
& +z^{4}\left[\frac{\mathrm{e}^{\left(4 \eta_{2}+6 \gamma_{2}^{2}\right)(T-t)}-1}{\left(2 \eta_{2}+5 \gamma_{2}^{2}\right)\left(4 \eta_{2}+6 \gamma_{2}^{2}\right)}-\frac{\mathrm{e}^{\left(2 \eta_{2}+\gamma_{2}^{2}\right)(T-t)}-1}{\left(2 \eta_{2}+5 \gamma_{2}^{2}\right)\left(2 \eta_{2}+\gamma_{2}^{2}\right)}\right] \\
& \left.+y^{2} z^{2} \frac{\left(\mathrm{e}^{\left(2 \eta_{1}+\gamma_{1}^{2}\right)(T-t)}-1\right)\left(\mathrm{e}^{\left(2 \eta_{2}+\gamma_{2}^{2}\right)(T-t)}-1\right)}{\left(2 \eta_{1}+\gamma_{1}^{2}\right)\left(2 \eta_{2}+\gamma_{2}^{2}\right)}\right] .
\end{aligned}
$$

\subsection{Application to the Heston model}

The model is defined by the following choices of coefficients

$$
f_{i}(v)=\sqrt{v} ; \quad \eta_{i}(v)=b_{i}\left(a_{i}-v\right) ; \quad \gamma_{i}(v)=c_{i} \sqrt{v}, \quad a_{i}, b_{i}, c_{i} \in \mathbb{R}^{+}, \quad i=1,2
$$


so that (2) and (3) become

$$
\begin{array}{ll}
d S_{t}^{1}=S_{t}^{1}\left(r d t+\sqrt{v_{t}^{1}} d W_{t}^{1}\right), & d v_{t}^{1}=b_{1}\left(a_{1}-v_{t}^{1}\right) d t+c_{1} \sqrt{v_{t}^{1}} d B_{t}^{1} \\
d S_{t}^{2}=S_{t}^{2}\left(r d t+\sqrt{v_{t}^{2}} d Z_{t}\right), & d v_{t}^{2}=b_{2}\left(a_{2}-v_{t}^{2}\right) d t+c_{2} \sqrt{v_{t}^{2}} d B_{t}^{2} .
\end{array}
$$

The Heston diffusion model is affine, in the sense of Duffie et al. (2000), in the state variables $\left(\log \left(S^{1}\right), \log \left(S^{2}\right), v^{1}, v^{2}\right)$ only when the correlation between the two assets, $\rho$, is zero. In this case, a semi-closed form for the price of a chooser (or exchange) option is available by the transform method (see Duffie et al. (2000)). Even though affinity is no longer preserved for different values of $\rho$, we may exploit this fact to compute more accurately a first term in the expansion, at correlations $(0, \beta, v)$ and then correct it with higher order terms relative only to $\rho$. This means that we are looking at the following dynamics for the three state processes

$$
\begin{aligned}
& d X_{t}=\sqrt{v_{t}^{1}} d \bar{W}_{t}^{1}-\sqrt{v_{t}^{2}} d \bar{W}_{t}^{2}-\frac{1}{2}\left(v_{t}^{1}+v_{t}^{2}\right) d t \\
& d v_{t}^{1}=b_{1}\left(a_{1}-v_{t}^{1}\right) d t+c_{1} \sqrt{v_{t}^{1}} d W_{t}^{3} \\
& d v_{t}^{2}=b_{2}\left(a_{2}-v_{t}^{2}\right) d t+c_{2} \sqrt{v_{t}^{2}} d W_{t}^{4} .
\end{aligned}
$$

with $\bar{W}^{1}, \bar{W}^{2}$ independent one dimensional Brownian motions and $<\bar{W}^{1}, W^{3}>_{t}=$ $\beta t$ and $<\bar{W}^{2}, W^{4}>_{t}=v t$.

By appropriate changes of probabilities to equivalent $Q_{1}$ and $Q_{2}$, the solution of the evaluation PDE (6), specialized for the model (32), may be written as

$$
\begin{aligned}
u_{0}(t, x, y, z) & =\mathrm{e}^{x} P_{1}(t, T, x, y, z)-P_{2}(t, T, x, y, z) \\
& =\mathrm{e}^{x} Q_{1}\left(X_{T}^{t, x, y, z} \geq 0\right)-Q_{2}\left(X_{T}^{t, x, y, z} \geq 0\right) .
\end{aligned}
$$

Substituting this functional form into (6), one finds that $P_{j}(t, T, x, y, z), j=1,2$, have to verify the following PDE's

$$
\begin{aligned}
& \frac{\partial P_{j}}{\partial t}+\frac{y+z}{2} \frac{\partial^{2} P_{j}}{\partial x^{2}}+\frac{c_{1}^{2} y}{2} \frac{\partial^{2} P_{j}}{\partial y^{2}}+\frac{c_{2}^{2} z}{2} \frac{\partial^{2} P_{j}}{\partial z^{2}}+v c_{1} y \frac{\partial^{2} P_{j}}{\partial x \partial y}-\beta c_{2} z \frac{\partial^{2} P_{j}}{\partial x \partial z} \\
& +\frac{(-1)^{j-1}(y+z)}{2} \frac{\partial P_{j}}{\partial x}+\left(b_{1} a_{1}-\bar{b}_{1, j} y\right) \frac{\partial P_{j}}{\partial y}+\left(b_{2} a_{2}-\bar{b}_{2, j} z\right) \frac{\partial P_{j}}{\partial z}=0
\end{aligned}
$$

where $\bar{b}_{1,1}=b_{1}-v c_{1}, \bar{b}_{1,2}=b_{1}, \bar{b}_{2,1}=b_{2}$ and $\bar{b}_{2,2}=b_{2}-\beta c_{2}$. FeynmanKaĉ formula gives that $P_{j}(t, T, x, y, z)=\mathbf{E}_{Q_{j}}\left(\mathbf{1}_{\left\{\tilde{X}_{T}^{j, t, x, y, z} \geq 0\right\}}\right)$, where

$$
\begin{aligned}
d \tilde{X}_{s}^{j, t, x, y, z} & =\sqrt{v_{s}^{j, 1, t, y}} d \tilde{W}_{s}^{1}-\sqrt{v_{s}^{j, 2, t, z}} d \tilde{W}_{s}^{2}+(-1)^{j-1}\left(v_{s}^{j, 1, t, y}+v_{s}^{j, 2, t, z}\right) d s \\
d v_{s}^{j, 1, t, y} & =\left(a_{1} b_{1}-\bar{b}_{1, j} v_{s}^{j, 1, t, y}\right) d s+c_{1} \sqrt{v_{s}^{j, 1, t, y}} d \tilde{W}_{s}^{3} \\
d v_{s}^{j, 2, t, z} & =\left(a_{2} b_{2}-\bar{b}_{2, j} v_{s}^{j, 2, t, z}\right) d s+c_{2} \sqrt{v_{s}^{j, 2, t, z}} d \tilde{W}_{s}^{4},
\end{aligned}
$$


with $<\tilde{W}^{1}, \tilde{W}^{2}>_{s}=0,<\tilde{W}^{1}, \tilde{W}^{3}>_{s}=v s$ and $<\tilde{W}^{2}, \tilde{W}^{4}>_{s}=\beta s$ all $s$.

These functions are eventually obtained by Fourier inversion

$$
P_{j}(t, T, x, y, z)=\frac{1}{2}+\frac{1}{\pi} \int_{0}^{+\infty} \Re\left\{\frac{f_{j}(\zeta, t, T, x, y, z)}{\mathrm{i} \zeta}\right\} d \zeta, \quad \mathrm{i}^{2}=-1
$$

and $f_{j}$ denote the joint characteristic functions of $\tilde{X}_{T}^{j, t, x, y, z}, v_{T}^{j, 1, t, y}, v_{T}^{j, 2, t, z}$. Those characteristic functions verify the same PDE as the $P_{j}$ 's with final condition $\mathrm{e}^{i x}$ and they are given explicitly by

$$
f_{j}(\zeta, t, T, x, y, z)=\exp \left(C_{j}(T-t)+D_{1, j}(T-t) y+D_{2, j}(T-t) z+\mathrm{i} x \zeta\right)
$$

where for $j=1,2$ the above functions are

$$
\begin{aligned}
D_{1, j}(T-t)= & \frac{\bar{b}_{1, j}-v c_{1} \mathrm{i} \zeta+d_{1, j}}{c_{1}^{2}} \frac{1-\mathrm{e}^{d_{1, j}(T-t)}}{1-g_{1, j} \mathrm{e}^{d_{1, j}(T-t)}}, \\
D_{2, j}(T-t)= & \frac{\bar{b}_{2, j}-\beta c_{2} \mathrm{i} \zeta+d_{2, j}}{c_{2}^{2}} \frac{1-\mathrm{e}^{d_{2, j}(T-t)}}{1-g_{2, j} \mathrm{e}^{d_{2, j}(T-t)}}, \\
C_{j}(T-t)= & \frac{a_{1} b_{1}}{c_{1}^{2}}\left[\left(\bar{b}_{1, j}-v c_{1} \mathrm{i} \zeta+d_{1, j}\right)(T-t)-2 \log \left(\frac{1-g_{1, j} \mathrm{e}^{d_{1, j}(T-t)}}{1-g_{1, j}}\right)\right] \\
& +\frac{a_{2} b_{2}}{c_{2}^{2}}\left[\left(\bar{b}_{2, j}-\beta c_{2} \mathrm{i} \zeta+d_{2, j}\right)(T-t)-2 \log \left(\frac{1-g_{2, j} \mathrm{e}^{d_{2, j}(T-t)}}{1-g_{2, j}}\right)\right], \\
g_{1, j}= & \frac{\bar{b}_{1, j}-v c_{1} \mathrm{i} \zeta+d_{1, j}}{\bar{b}_{1, j}-v c_{1} \mathrm{i} \zeta-d_{1, j}}, \quad g_{2, j}=\frac{\bar{b}_{2, j}-\beta c_{2} \mathrm{i} \zeta+d_{2, j}}{\bar{b}_{2, j}-\beta c_{2} \mathrm{i} \zeta-d_{2, j}} \\
d_{1, j}= & \sqrt{\left(\nu c_{1} \mathrm{i} \zeta-\bar{b}_{1, j}\right)^{2}+c_{1}^{2}(-1)^{j}\left(\mathrm{i} \zeta-\zeta^{2}\right)} \\
d_{1, j}= & \sqrt{\left(\beta c_{2} \mathrm{i} \zeta-\bar{b}_{2, j}\right)^{2}+c_{2}^{2}(-1)^{j}\left(\mathrm{i} \zeta-\zeta^{2}\right)},
\end{aligned}
$$

which are of very easy implementation.

To compute the first order terms, following our procedure, we recall that all the coefficients are written at correlations $(0,0,0)$, in other words we are looking at the Markovian triple described by (32), but with independent Brownian motions, and the mean volatility is given by

$$
\Sigma_{T}^{t, y, z}=\int_{t}^{T}\left(v_{s}^{1, t, y}+v_{s}^{2, t, z}\right) d s
$$


whose average can be computed explicitly

$$
\begin{aligned}
\bar{\Sigma}_{T}^{t, y, z} & =\int_{t}^{T} \mathbf{E}^{*}\left(v_{s}^{1, t, y}+v_{s}^{2, t, z}\right) d s \\
& =\left(a_{1}+a_{2}\right)(T-t)+\frac{y-a_{1}}{b_{1}}\left(1-\mathrm{e}^{-b_{1}(T-t)}\right)+\frac{z-a_{2}}{b_{2}}\left(1-\mathrm{e}^{-b_{2}(T-t)}\right),
\end{aligned}
$$

since $\mathbf{E}^{*}\left(v_{s}^{i, t, w_{i}}\right)=\left(w_{i}-a_{i}\right) \mathrm{e}^{-b_{i}(s-t)}+a_{i}$ for $i=1,2$.

Looking at the formula for the coefficient (18) and its approximation (24), we may conclude

$$
\begin{aligned}
& u_{1}(t, x, y, z)=-\mathbf{E}^{*}\left[\frac{\mathcal{N}^{\prime}\left(d_{2}\left(x, \Sigma_{T}^{t, y, z}\right)\right)}{\sqrt{\Sigma_{T}^{t, y, z}}} \int_{t}^{T} \sqrt{v_{\alpha}^{1, t, y} v_{\alpha}^{2, t, y}} d \alpha\right] \\
& \bar{u}_{1}(t, x, y, z)=-\frac{\mathcal{N}^{\prime}\left(\bar{d}_{2}\right)}{\sqrt{\bar{\Sigma}_{T}^{t, y, z}}} \int_{t}^{T} \mathbf{E}^{*}\left[\sqrt{v_{\alpha}^{1, t, y}}\right] \mathbf{E}^{*}\left[\sqrt{v_{\alpha}^{2, t, y}}\right] d \alpha,
\end{aligned}
$$

where we used the independence between $v^{1}$ and $v^{2}$.

It remains to compute $\mathbf{E}^{*}\left[\sqrt{v_{\alpha}^{i, t, w_{i}}}\right]$.

For $i=1,2$, we know that $v_{\alpha}^{i, t, w_{i}}$ follows a non central chi-squared distribution with density given by

$$
g_{i}(\xi)=C_{i} \mathrm{e}^{-A_{i}-C_{i} \xi}\left(\frac{C_{i} \xi}{A_{i}}\right)^{q_{i} / 2} I_{q_{i}}\left(2 \sqrt{A_{i} C_{i} \xi}\right)
$$

where

$$
C_{i}=\frac{2 b_{i}}{c_{i}^{2}\left(1-\mathrm{e}^{-b_{i}(\alpha-t)}\right)}, \quad A_{i}=C_{i} w_{i} \mathrm{e}^{-b_{i}(\alpha-t)}, \quad q_{i}=\frac{2 a_{i} b_{i}}{c_{i}^{2}}-1
$$

and $I_{q}(\cdot)$ is the modified Bessel function of the first kind of order $q$

$$
I_{q}(\zeta)=\left(\frac{\zeta}{2}\right)^{q} \sum_{k=0}^{\infty}\left(\frac{\zeta^{2}}{4}\right)^{k} \frac{1}{k ! \Gamma(k+q+1)}
$$

Therefore, to compute the above expected value, we have

$$
\mathbf{E}^{*}\left[\sqrt{v_{\alpha}^{i, t, w_{i}}}\right]=\int_{0}^{\infty} \sqrt{\xi} g_{i}(\xi) d \xi
$$




$$
\begin{aligned}
= & C_{i}\left(\frac{C_{i}}{A_{i}}\right)^{q_{i} / 2}\left(A_{i} C_{i}\right)^{q_{i} / 2} \mathrm{e}^{-A_{i}} \\
& \times \sum_{k=0}^{\infty} \frac{\left(A_{i} C_{i}\right)^{k}}{k ! \Gamma\left(k+q_{i}+1\right)} \int_{0}^{+\infty} \xi^{q_{i}+k+1 / 2} \mathrm{e}^{-C_{i} \xi} d \xi \\
= & \left(C_{i}\right)^{q_{i}+1} \mathrm{e}^{-A_{i}} \sum_{k=0}^{\infty} \frac{\left(A_{i} C_{i}\right)^{k}}{k ! \Gamma\left(k+q_{i}+1\right)} \int_{0}^{+\infty} \xi^{q_{i}+k+1 / 2} \mathrm{e}^{-C_{i} \xi} d \xi \\
= & \frac{\mathrm{e}^{-A_{i}}}{\sqrt{C_{i}}} \sum_{k=0}^{\infty} \frac{A_{i}^{k} \Gamma\left(k+q_{i}+3 / 2\right)}{\Gamma\left(k+q_{i}+1\right)} .
\end{aligned}
$$

where we used the substitution $\mu=C_{i} \xi$ in the last integral.

Plugging back the specifications (36) into this last expression and denoting by $\lambda_{i}(s-t):=\frac{\mathrm{e}^{-b_{i}(\alpha-t)}}{1-\mathrm{e}^{-b_{i}(\alpha-t)}}$, we arrive at the final expression for the expectation

$$
\begin{aligned}
\mathbf{E}^{*}\left[\sqrt{\left.v_{\alpha}^{i, t, w_{i}}\right]=}\right. & {\left[\frac{c_{i}^{2}\left(1-\mathrm{e}^{-b_{i}(\alpha-t)}\right)}{2 b_{i}}\right]^{1 / 2} } \\
& \times \mathrm{e}^{-\frac{2 b_{i} y}{c_{i}^{2}} \lambda_{i}(\alpha-t)} \sum_{k=0}^{\infty}\left(\frac{2 b_{i} w_{i}}{c_{i}^{2}}\right)^{k} \lambda_{i}(\alpha-t)^{k} \frac{\Gamma\left(q_{i}+k+3 / 2\right)}{k ! \Gamma\left(k+q_{i}+1\right)}
\end{aligned}
$$

From the above computations it follows

$$
\begin{aligned}
\bar{u}_{1}(t, x, y, z)= & -\frac{c_{1} c_{2} \mathcal{N}^{\prime}\left(\bar{d}_{2}\right)}{2 \sqrt{b_{1} b_{2} \bar{\Sigma}_{T}^{t, y, z}}} \sum_{k=0}^{+\infty} \sum_{j=0}^{+\infty} \\
& \times\left[\frac{2 b_{1} y}{c_{1}^{2}}\right]^{k} \frac{\Gamma\left(q_{1}+k+3 / 2\right)}{k ! \Gamma\left(k+q_{1}+1\right)}\left[\frac{2 b_{2} z}{c_{2}^{2}}\right]^{j} \frac{\Gamma\left(q_{2}+j+3 / 2\right)}{j ! \Gamma\left(j+q_{2}+1\right)} \\
& \times \int_{t}^{T} \frac{\mathrm{e}^{-\frac{2 b_{1} y}{c_{1}^{2}} \lambda_{1}(\alpha-t)-\frac{2 b_{2} y}{c_{2}^{2}} \lambda_{2}(\alpha-t)}}{\mathrm{e}^{\frac{\left(b_{1}+b_{2}\right)}{2}(\alpha-t)}} \lambda_{1}(\alpha-t)^{k-\frac{1}{2}} \lambda_{2}(\alpha-t)^{j-\frac{1}{2}} d \alpha .
\end{aligned}
$$

This term is computationally quite engaging, as we need to estimate accurately the double series appearing above, but the implementation remains manageable.

A simpler situation occurs for the two terms with respect to $v$ and $\beta$. From (19), we have

$\phi_{1}(t, x, y, z)=-\int_{t}^{T} \int_{\alpha}^{T} \mathbf{E}^{*}\left[c_{1} v_{\alpha}^{1, t, y} \exp \left\{-\int_{\alpha}^{\alpha_{1}}\left(b_{1}+\frac{c_{1}^{2}}{8 v_{\alpha_{2}}^{1, t, y}}\right) d u\right.\right.$ 


$$
\left.\left.+\frac{c_{1}}{2} \int_{\alpha}^{\alpha_{1}} \frac{1}{\sqrt{v_{\alpha_{2}}^{1, t, y}}} d W_{\alpha_{2}}^{3}\right\} \times \frac{\mathcal{N}^{\prime}\left(d_{2}\left(x, \Sigma_{T}^{t, y, z}\right)\right) d_{2}\left(x, \Sigma_{T}^{t, y, z}\right)}{\Sigma_{T}^{t, y, z}}\right] d \alpha_{1} d \alpha .
$$

Since $\exp \left\{-\int_{\alpha}^{\alpha_{1}}\left(b_{1}+\frac{c_{1}^{2}}{8 v_{\alpha_{2}}^{1, t, y}}\right) d u+\frac{c_{1}}{2} \int_{\alpha}^{\alpha_{1}} \frac{1}{\sqrt{v_{\alpha_{2}}^{1, t, y}}} d W_{\alpha_{2}}^{3}\right\}$ is an exponential martingale starting at $\alpha$ with initial condition 1, the approximation (25) of $\phi_{1}$ becomes

$$
\begin{aligned}
\bar{\phi}_{1}(t, x, y, z)= & -\frac{\mathcal{N}^{\prime}\left(\bar{d}_{2}\right) \bar{d}_{2}}{\bar{\Sigma}_{T}^{t, y, z}} \int_{t}^{T} \int_{\alpha}^{T} \mathbf{E}^{*}\left[c_{1} v_{\alpha}^{1, t, y} \mathrm{e}^{-b_{1}\left(\alpha_{1}-\alpha\right)}\right] d \alpha_{1} d \alpha \\
= & -\frac{\mathcal{N}^{\prime}\left(\bar{d}_{2}\right) \bar{d}_{2}}{\bar{\Sigma}_{T}^{t, y, z}} \int_{t}^{T} \int_{\alpha}^{T} c_{1}\left(\left(y-a_{1}\right) \mathrm{e}^{-b_{1}(\alpha-t)}+a_{1}\right) \mathrm{e}^{-b_{1}\left(\alpha_{1}-\alpha\right)} d \alpha_{1} d \alpha \\
= & -\frac{c_{1} \mathcal{N}^{\prime}\left(\bar{d}_{2}\right) \bar{d}_{2}}{b_{1} \bar{\Sigma}_{T}^{t, y, z}}\left[\frac{y-2 a_{1}}{b_{1}}\left(1-\mathrm{e}^{-b_{1}(T-t)}\right)\right. \\
& \left.+(T-t)\left(a_{1}-\left(y-a_{1}\right) \mathrm{e}^{-b_{1}(T-t)}\right)\right] .
\end{aligned}
$$

Similarly, from (26), we obtain

$$
\begin{aligned}
\bar{\psi}_{1}(t, x, y, z)= & \frac{c_{2} \mathcal{N}^{\prime}\left(\bar{d}_{2}\right) \bar{d}_{1}}{b_{2} \bar{\Sigma}_{T}^{t, y, z}}\left[\frac{z-2 a_{2}}{b_{2}}\left(1-\mathrm{e}^{-b_{2}(T-t)}\right)\right. \\
& \left.+(T-t)\left(a_{2}-\left(z-a_{2}\right) \mathrm{e}^{-b_{2}(T-t)}\right)\right] .
\end{aligned}
$$

\subsection{Numerical results}

In this section we report some numerical results obtained by implementing the approximation formulas introduced in the previous sections for the Hull and White and the Heston models. Without loss of generality, in all experiments we set $t=0$.

For the Hull and White model we compare our approximation with the Monte Carlo price, that we denote $\hat{u}_{M C}$.

Even though the Monte Carlo procedure for this model is rather straightforward, its computational time remains rather lengthy, while our method requires a time comparatively negligible.

For the Heston case things are a little different. As long as we remain in the affine case $(0, \beta, v)$ we may compute the term (33) by numerical inversion of the Fourier transform, while in the non-affine case $\rho \neq 0$ again we have to resort to the Monte Carlo price to define a benchmark for our method. The simulation of the Heston model is much more involved than for the Hull and White dynamics, due to the structure of the CIR diffusion equations. Many different schemes have been proposed in recent 
Table 1 Differences $\tilde{u}_{0}-\hat{u}_{\mathrm{MC}}$ for the Hull and White model

\begin{tabular}{lrrr}
\hline$T \backslash S_{0}^{1} / S_{0}^{2}$ & $100 / 95$ & $100 / 100$ & $100 / 105$ \\
\hline$T=0.25$ & -0.0139 & 0.0069 & -0.0006 \\
$T=0.5$ & -0.0060 & -0.0020 & -0.0085 \\
$T=1$ & -0.0061 & 0.0056 & 0.0209 \\
\hline
\end{tabular}

All the approximated prices fall within the $95 \%$ confidence interval of the Monte Carlo estimates. The estimated standard deviations of $\hat{u}_{\mathrm{MC}}$ range from 0.0075 to 0.0240

Table $2 T=.25$

\begin{tabular}{lllr}
\hline$\rho \backslash S_{0}^{1} / S_{0}^{2}$ & $100 / 95$ & $100 / 100$ & $100 / 105$ \\
\hline-0.5 & 0.0660 & 0.0736 & 0.0470 \\
-0.3 & 0.0329 & 0.0397 & $\mathbf{0 . 0 0 6 0}$ \\
-0.1 & $\mathbf{0 . 0 2 1 0}$ & $\mathbf{0 . 0 0 6 6}$ & $-\mathbf{0 . 0 0 8 6}$ \\
0 & $\mathbf{0 . 0 2 1 7}$ & $\mathbf{0 . 0 1 0 2}$ & $-\mathbf{0 . 0 1 0 4}$ \\
0.1 & $\mathbf{0 . 0 1 7 3}$ & $\mathbf{0 . 0 0 8 8}$ & $\mathbf{0 . 0 0 7 5}$ \\
0.3 & $\mathbf{0 . 0 1 1 6}$ & 0.0472 & 0.0261 \\
0.5 & 0.0612 & 0.1244 & 0.0667 \\
\hline
\end{tabular}

literature (see e.g. Lord et al. 2006; Andersen 2008 and the references therein). The exact and bias-free technique in Broadie and Kaya (2006) does not appear to extend easily to our model, due to the correlation structure. Therefore we resorted to an EulerMaruyama discretization scheme. It is well known that a näive implementation of this scheme can produce negative samples of the variance process even for values of the parameters satisfying the Feller condition $c^{2}<2 k \theta$. Different solutions have been proposed to get around this problem. In our experiments we chose to implement the scheme denoted full truncation, as it appears to produce the smallest discretization bias among a set of alternative schemes (see Lord et al. 2006).

Results for the Hull and White model. In this paragraph we test the performance of our approximation technique for the Hull and White model. For our experiment we fixed the values of the parameters $\beta$ and $v$ (around typical market values) while varying the values of the asset-to-asset correlation $\rho$, the ratio $S_{0}^{1} / S_{0}^{2}$ and the maturity $T$. We used formula (28) with the coefficients developed in Sect. 4.1.

The model parameters were set to $\eta_{1}=\eta_{2}=0, \gamma_{1}=0.1, \gamma_{2}=0.15$ and the asset-to-volatility correlations were $\beta=-0.45$ and $v=-0.55$. The Monte Carlo estimates of the prices $\hat{u}_{M C}$ were obtained by simulating $M=250000$ paths with $N=1000$ equispaced time-grid points on the interval $[0, T]$ needed for the numerical evaluation of the integrals involved in the solution of (29).

In the first table (Table 1) we report the difference between the price computed by using the second-order approximation (27) of $u_{0}$ and the Monte Carlo estimate $\hat{u}_{M C}$ with $\rho=\beta=v=0$. The results show the quality of such an approximation.

Next we fixed $\beta$ and $\nu$ to the chosen market values and we let $\rho$ and the ratio $S_{0}^{1} / S_{0}^{2}$ vary. The next three tables show the difference between our approximation and the 
Table $3 \quad T=.5$

\begin{tabular}{lrrr}
\hline$\rho \backslash S_{0}^{1} / S_{0}^{2}$ & $100 / 95$ & $100 / 100$ & $100 / 105$ \\
\hline-0.5 & 0.1051 & 0.0934 & 0.0805 \\
-0.3 & 0.0607 & $\mathbf{0 . 0 2 2 4}$ & $\mathbf{0 . 0 2 7 5}$ \\
-0.1 & $\mathbf{0 . 0 0 9 6}$ & $\mathbf{0 . 0 2 2 7}$ & $\mathbf{0 . 0 0 6 6}$ \\
0 & $\mathbf{- 0 . 0 1 2 1}$ & $\mathbf{0 . 0 0 1 0}$ & $\mathbf{- 0 . 0 0 8 9}$ \\
0.1 & $\mathbf{0 . 0 1 5 7}$ & $\mathbf{- 0 . 0 0 4 1}$ & $\mathbf{0 . 0 0 2 5}$ \\
0.3 & 0.0347 & 0.0730 & 0.0432 \\
0.5 & 0.1175 & 0.1759 & 0.1394 \\
\hline
\end{tabular}

Table $4 \quad T=1$

\begin{tabular}{lllr}
\hline$\rho \backslash S_{0}^{1} / S_{0}^{2}$ & $100 / 95$ & $100 / 100$ & $100 / 105$ \\
\hline-0.5 & 0.1287 & 0.1453 & 0.1747 \\
-0.3 & 0.0862 & 0.0571 & 0.0759 \\
-0.1 & $\mathbf{0 . 0 2 9 4}$ & $\mathbf{0 . 0 0 4 4}$ & $-\mathbf{0 . 0 3 1 4}$ \\
0 & $\mathbf{0 . 0 3 5 9}$ & 0.0440 & $\mathbf{- 0 . 0 0 3 7}$ \\
0.1 & $\mathbf{0 . 0 1 8 2}$ & $\mathbf{0 . 0 0 9 6}$ & $\mathbf{- 0 . 0 0 4 1}$ \\
0.3 & 0.0761 & 0.0926 & 0.0657 \\
0.5 & 0.1796 & 0.2529 & 0.2031 \\
\hline
\end{tabular}

Monte Carlo estimate for $T=0.25$ (Table 2), $T=0.5$ (Table 3) and $T=1$ (Table 4). We marked the results in bold when the approximated price lied in the (estimated) $95 \%$ confidence interval. The estimated standard deviations of $\hat{u}_{M C}$ range from 0.0046 to 0.0159 for $T=0.25$, from 0.0080 to 0.0213 for $T=0.5$ and from 0.0129 to 0.0291 for $T=1$. For large values of the correlation parameter $\rho(|\rho| \geq .3)$ the approximation improves when $T$ becomes smaller (with the only exception $\rho=-0.3$ and $S_{0}^{1} / S_{0}^{2}=$ $100 / 100)$. For $|\rho| \leq .1$ the errors range from $0.001\left(\rho=0, S_{0}^{1} / S_{0}^{2}=100 / 100\right.$ and $T=0.5)$ to $0.0440\left(\rho=0, S_{0}^{1} / S_{0}^{2}=100 / 100\right.$ and $\left.T=1\right)$.

Results for the Heston model We first considered the affine case $(\rho=0)$ for the Heston model, since in this context we can compare our approximation with the exact price, evaluated according to (33). The model parameters were fixed to $a_{1}=0.025$, $a_{2}=0.035, b_{1}=1.6, b_{2}=1.1, c_{1}=0.45, c_{2}=0.4, y=0.025, z=0.035$, according to typical market values, as found in the literature (see e.g. Bakshi et al. 1997). Table 5 reports the errors for varying values of $(\beta, v)$ and $\left(T, S_{0}^{1} / S_{0}^{2}\right)$. As expected, we attain the best results for small values of the correlations $\beta$ and $\nu$. The errors tend to become larger also as $T$ increases. In the small correlation scenarios, the absolute errors are smaller for the out-of-money options than for the in-the-money options, at each maturity. This monotonicity with respect to the starting values $S_{0}^{1} / S_{0}^{2}$ gets lost at larger correlations. Finally, our approximation seems to overestimate the true value when correlations are small $[(\beta, v)=(-0.1,-0.1)]$ and to underestimate it when correlations are large $((\beta, v)=(-0.7,-0.7))$.

Netx, we considered the non-affine case $\rho \neq 0$. The asset-to-volatility correlations were set to $v=-0.6$ and $\beta=-0.76$ (see Bakshi et al. (1997)). The corresponding Monte Carlo prices were obtained by simulating $M=6,40,000$ paths from the SDE (31) by means of the full-truncation Euler-Maruyama scheme with 800 equispaced time-grid points on the interval $[0, T]$. The estimated standard deviations of the Monte 
Table 5 Errors and (absolute relative errors) for the Heston model in the affine case $\rho=0$

\begin{tabular}{llrrr}
\hline & $(-01,-0.1)$ & $(-0.3-0.3)$ & \multicolumn{1}{c}{$(-05,-0.5)$} & \multicolumn{1}{c}{$(-0.7,-0.7)$} \\
\hline$(0.25,100 / 95)$ & $0.0075(0.0010)$ & $0.0129(0.0017)$ & $0.0039(0.0005)$ & $-0.0225(0.0030)$ \\
$(0.25,100 / 100)$ & $0.0038(0.0008)$ & $-0.0014(0.0003)$ & $-0.0250(0.0053)$ & $-0.0705(0.0153)$ \\
$(0.25,100 / 105)$ & $0.0003(0.0001)$ & $-0.0085(0.0031)$ & $-0.0292(0.0105)$ & $-0.0603(0.0220)$ \\
$(0.5,100 / 95)$ & $0.0168(0.0018)$ & $0.0271(0.0029)$ & $0.0023(0.0003)$ & $-0.0669(0.0075)$ \\
$(0.5,100 / 100)$ & $0.0111(0.0017)$ & $0.0064(0.0010)$ & $-0.0376(0.0058)$ & $-0.1297(0.0205)$ \\
$(0.5,100 / 105)$ & $0.0054(0.0012)$ & $-0.0069(0.0015)$ & $-0.0499(0.0111)$ & $-0.1241(0.0282)$ \\
$(1,100 / 95)$ & $0.0393(0.0033)$ & $0.0736(0.0063)$ & $0.0432(0.0038)$ & $-0.0683(0.0061)$ \\
$(1,100 / 100)$ & $0.0297(0.0032)$ & $0.0414(0.0045)$ & $-0.0156(0.0017)$ & $-0.1565(0.0181)$ \\
$(1,100 / 105)$ & $0.0198(0.0027)$ & $0.0147(0.0021)$ & $-0.0515(0.0074)$ & $-0.1858(0.0275)$ \\
\hline
\end{tabular}

Several scenarios are considered with varying maturities and starting values $\left(T, S_{0}^{1} / S_{0}^{2}\right)$ (the rows) and asset-to-volatility correlation levels $(\beta, v)$ (the columns)

Table 6 Errors obtained with the approximations (37) and (28) (in parenthesis) for $T=.25$

\begin{tabular}{lrrr}
\hline$\rho \backslash S_{0}^{1} / S_{0}^{2}$ & \multicolumn{1}{c}{$100 / 95$} & $100 / 100$ & \multicolumn{1}{c}{$100 / 105$} \\
\hline-0.3 & $\mathbf{0 . 0 0 8 5}(-0.0856)$ & $0.0785(0.1002)$ & $0.0733(0.1877)$ \\
-0.1 & $-\mathbf{0 . 0 0 5 2}(-0.0825)$ & $\mathbf{0 . 0 0 4 6}(0.0547)$ & $0.0409(0.1740)$ \\
0.1 & $\mathbf{0 . 0 1 9 7 ( - 0 . 0 4 0 9 )}$ & $\mathbf{0 . 0 0 5 6}(0.0841)$ & $-0.0199(0.1317)$ \\
0.3 & $0.0471(-0.0219)$ & $0.0218(0.0861)$ & $-\mathbf{0 . 0 0 4 9}(0.1374)$ \\
\hline
\end{tabular}

Table 7 Errors obtained with the approximations (37) and (28) (in parenthesis) for $T=.5$

\begin{tabular}{llrr}
\hline$\rho \backslash S_{0}^{1} / S_{0}^{2}$ & $100 / 95$ & $100 / 100$ & \multicolumn{1}{c}{$100 / 105$} \\
\hline-0.3 & $\mathbf{0 . 0 2 3 7}(-\mathbf{0 . 0 3 8 9})$ & $0.0342(0.1479)$ & $0.0811(0.3065)$ \\
-0.1 & $\mathbf{0 . 0 0 9 1}(-0.0535)$ & $\mathbf{0 . 0 0 6 4}(0.1200)$ & $0.0239(0.2493)$ \\
0.1 & $\mathbf{0 . 0 0 7 3}(-0.0552)$ & $-\mathbf{0 . 0 0 7 8}(0.1058)$ & $0.0278(0.2531)$ \\
0.3 & $0.0957(0.0431)$ & $0.0909(0.2045)$ & $\mathbf{0 . 0 1 8 0}(0.2434)$ \\
\hline
\end{tabular}

Carlo prices $\hat{u}_{M C}$ range from 0.0079 to 0.0171 for $T=0.25$, from 0.0092 to 0.0148 for $T=0.5$ and from 0.0133 to 0.0317 for $T=1$.

In this case, the results (Table 6 in parenthesis) are not completely satisfactory, since the approximation error ranges from -0.0219 to 0.2951 for in-the-money and at-the-money options and even higher for the out-the-money case. This behavior would suggest to push the approximation further than the first order (see the "Appendix") for the Heston model. This additional computational effort may be avoided, as we noticed that we achieve much better results by using the following first order approximation

$$
u(t, x, y, z ; \rho, v, \beta) \approx u(t, x, y, z ; 0, \beta, v)+\rho \bar{u}_{1}(t, x, y, z) .
$$

Indeed, by exploiting the exact numerical evaluation of the 0 -th term $u(t, x, y, z ; 0, \beta$, $v)$, this approximation captures the apparently strong non-linearity in the correlations $(\beta, \nu)$ (see Tables 6, 7, 8).

Acknowledgments We would like to thank the referee for useful comments and pointing us out ref. Bakshi and Madan (2000). 
Table 8 Errors obtained with the approximations (37) and (28) (in parenthesis) for $T=1$

\begin{tabular}{lrrr}
\hline$\rho \backslash S_{0}^{1} / S_{0}^{2}$ & \multicolumn{1}{c}{$100 / 95$} & $100 / 100$ & \multicolumn{1}{c}{$100 / 105$} \\
\hline-0.3 & $\mathbf{0 . 0 2 9 4}(-0.0681)$ & $\mathbf{0 . 0 2 9 0}(0.1497)$ & $0.0702(0.3540)$ \\
-0.1 & $-0.0475(-0.1451)$ & $\mathbf{0 . 0 1 7 8}(0.1386)$ & $-\mathbf{0 . 0 0 1 9}(0.2819)$ \\
0.1 & $\mathbf{0 . 0 1 1 9}(-0.0857)$ & $0.0361(0.1568)$ & $\mathbf{0 . 0 0 5 4}(0.2892)$ \\
0.3 & $0.1531(0.0556)$ & $0.1478(0.2686)$ & $0.1015(0.3852)$ \\
\hline
\end{tabular}

\section{Appendix}

In this appendix we want to show briefly how the second order terms may be computed with our technique.

Referring to the evaluation PDE (6), by differentiating it twice with respect to the three correlation parameters one obtains the following chain of PDE problems for $x \in \mathbb{R}, y, z>0$ and $t \in[0, T]$

$$
\begin{aligned}
& \left\{\begin{array}{l}
\mathcal{L}_{0} u_{0}(t, x, y, z)=0 \\
u_{0}(T, x, y, z)=\left(\mathrm{e}^{x}-1\right)^{+}
\end{array}\right. \\
& \left\{\begin{array}{l}
\mathcal{L}_{0} \phi_{1}=-\mathcal{B} u_{0} \\
\phi_{1}(T, x, y, z)=0
\end{array}\right. \\
& \left\{\begin{array}{l}
\mathcal{L}_{0} u_{2}=\mathcal{A} u_{1} \\
u_{2}(T, x, y, z)=0 .
\end{array}\right. \\
& \left\{\begin{array}{l}
\mathcal{L}_{0} \psi_{2}=-\mathcal{G} \psi_{1} \\
u_{1}(T, x, y, z)=0 .
\end{array}\right. \\
& \psi_{2}(T, x, y, z)=0 \\
& \left\{\begin{array}{l}
\mathcal{L}_{0} \psi_{1}=\mathcal{G} u_{0} \\
\psi_{1}(T, x, y, z)=0 .
\end{array}\right. \\
& \left\{\begin{array}{l}
\mathcal{L}_{0} \theta_{13}=\mathcal{A} \psi_{1}+\mathcal{G} u_{1}-\mathcal{A}_{2} u_{0} \phi_{2}=-\mathcal{B} \phi_{1} \\
\theta_{13}(T, x, y, z)=0
\end{array}\right.
\end{aligned}
$$

Hence, by using again Duhamel's principle and Feynman-Kac's representation of the solutions of the PDE's, we obtain that

$$
\begin{aligned}
& u_{2}(t, x, y, z)=-\int_{t}^{T} \mathbf{E}\left((\mathcal{A} u)\left(\alpha, X_{\alpha}^{t, x, y, z}, v_{\alpha}^{1, t, y}, v_{\alpha}^{2, t, z}\right)\right) d \alpha \\
& \phi_{2}(t, x, y, z)=-\int_{t}^{T} \mathbf{E}\left(\left(-\mathcal{B} \phi_{1}\right)\left(\alpha, X_{\alpha}^{t, x, y, z}, v_{\alpha}^{1, t, y}, v_{\alpha}^{2, t, z}\right)\right) d \alpha \\
& \psi_{2}(t, x, y, z)=-\int_{t}^{T} \mathbf{E}\left(\left(-\mathcal{G} \psi_{1}\right)\left(\alpha, X_{\alpha}^{t, x, y, z}, v_{\alpha}^{1, t, y}, v_{\alpha}^{2, t, z}\right)\right) d \alpha \\
& \theta_{12}(t, x, y, z)=-\int_{t}^{T} \mathbf{E}\left(\left(\mathcal{A} \phi_{1}-\mathcal{B} u_{1}+\mathcal{A}_{1} u_{0}\right)\left(\alpha, X_{\alpha}^{t, x, y, z}, v_{\alpha}^{1, t, y}, v_{\alpha}^{2, t, z}\right)\right) d \alpha
\end{aligned}
$$




$$
\begin{aligned}
& \theta_{13}(t, x, y, z)=-\int_{t}^{T} \mathbf{E}\left(\left(\mathcal{A} \psi_{1}+\mathcal{G} u_{1}-\mathcal{A}_{2} u_{0}\right)\left(\alpha, X_{\alpha}^{t, x, y, z}, v_{\alpha}^{1, t, y}, v_{\alpha}^{2, t, z}\right)\right) d \alpha \\
& \theta_{23}(t, x, y, z)=-\int_{t}^{T} \mathbf{E}\left(\left(-\mathcal{B} \psi_{1}+\mathcal{G} \phi_{1}\right)\left(\alpha, X_{\alpha}^{t, x, y, z}, v_{\alpha}^{1, t, y}, v_{\alpha}^{2, t, z}\right)\right) d \alpha
\end{aligned}
$$

and repeating the same calculations as before, we arrive at the explicit representation of those coefficients. The first three coefficients are obtained by iterating the corresponding first order coefficients

$$
\begin{aligned}
& u_{2}(t, x, y, z)=\mathbf{E}\left\{\frac{\left(d_{1} d_{2}-1\right) \mathcal{N}^{\prime}\left(d_{2}\right)}{\left(\Sigma_{T}^{t, y, z}\right)^{\frac{3}{2}}}\left(x, \Sigma_{T}^{t, y, z}\right)\right. \\
& \left.\times \int_{t}^{T} f_{1}\left(v_{\alpha}^{1, t, y}\right) f_{2}\left(v_{\alpha}^{2, t, z}\right) F_{[\alpha, T]}^{t, y, z} d \alpha\right\} \\
& \phi_{2}(t, x, y, z)=-\mathbf{E}\left\{\frac{\left(1-d_{2}^{2}\right) \mathcal{N}^{\prime}\left(d_{2}\right)}{\left(\Sigma_{T}^{t, y, z}\right)^{\frac{3}{2}}}\left(x, \Sigma_{T}^{t, y, z}\right)\right. \\
& \times \int_{t}^{T}\left(\gamma_{1} f_{1}\right)\left(v_{\alpha}^{1, t, y}\right)\left[\frac{\partial v_{\alpha}^{1, t, y}}{\partial y}\right]^{-2} \frac{\partial c_{[\alpha, T]}^{1, t, y}}{\partial y} d \alpha \\
& +\frac{\left(3 d_{2}^{2}-3+3 d_{1} d_{2}-d_{1} d_{2}^{3}\right) \mathcal{N}^{\prime}\left(d_{2}\right)}{2\left(\Sigma_{T}^{t, y, z}\right)^{\frac{5}{2}}}\left(x, \Sigma_{T}^{t, y, z}\right) \\
& \left.\int_{t}^{T}\left(\gamma_{1} f_{1}\right)\left(v_{\alpha}^{1, t, y}\right)\left[\frac{\partial v_{\alpha}^{1, t, y}}{\partial y}\right]^{-2} c_{[\alpha, T]}^{1, t, y} \frac{\partial \Sigma_{[\alpha, T]}^{t, y, z}}{\partial y} d \alpha\right\} \\
& \psi_{2}(t, x, y, z)=\mathbf{E}\left\{\frac{\left(1-d_{1}^{2}\right) \mathcal{N}^{\prime}\left(d_{2}\right)}{\left(\Sigma_{T}^{t, y, z}\right)^{\frac{3}{2}}}\left(x, \Sigma_{T}^{t, y, z}\right)\right. \\
& \left.\times \int_{t}^{T}\left(\gamma_{2} f_{2}\right)\left(v_{\alpha}^{2, t, z}\right)\right)\left[\frac{\partial v_{\alpha}^{2, t, z}}{\partial z}\right]^{-2} \frac{\partial c_{[\alpha, T]}^{2, t, z}}{\partial z} d \alpha
\end{aligned}
$$




$$
\begin{aligned}
& +\frac{\left(3 d_{1}^{2}-3+3 d_{1} d_{2}-d_{1}^{3} d_{2}\right) \mathcal{N}^{\prime}\left(d_{2}\right)}{2\left(\Sigma_{T}^{t, y, z}\right)^{\frac{5}{2}}}\left(x, \Sigma_{T}^{t, y, z}\right) \\
& \left.\times \int_{t}^{T}\left(\gamma_{2} f_{2}\right)\left(v_{\alpha}^{2, t, z}\right)\left[\frac{\partial v_{\alpha}^{2, t, z}}{\partial z}\right]^{-2} c_{[\alpha, T]}^{2, t, z} \frac{\partial \Sigma_{[\alpha, T]}^{t, y, z}}{\partial z} d \alpha\right\},
\end{aligned}
$$

while the coefficients coming from the mixed derivatives are

$$
\begin{aligned}
& \theta_{12}(t, x, y, z)=-\mathbf{E}\left[\frac{\left(2 d_{2}+d_{1}-d_{2}^{2} d_{1}\right) \mathcal{N}^{\prime}\left(d_{2}\right)}{2\left(\Sigma_{T}^{t, y, z}\right)^{2}}\left(x, \Sigma_{T}^{t, y, z}\right)\right. \\
& \times \int_{t}^{T}\left\{2 f_{1}\left(v_{\alpha}^{1, t, y}\right) f_{2}\left(v_{\alpha}^{2, t, z}\right) c_{[\alpha, T]}^{1, t, y}\right. \\
& \left.+\left(\gamma_{1} f_{1}\right)\left(v_{\alpha}^{1, t, y}\right) F_{[\alpha, T]}^{t, y, z} \frac{\partial \Sigma_{[\alpha, T]}^{t, y, z}}{\partial y}\right\}\left[\frac{\partial v_{\alpha}^{1, t, y}}{\partial y}\right]^{-1} d \alpha \\
& -\frac{\mathcal{N}^{\prime}\left(d_{2}\right)}{\Sigma_{T}^{t, y, z}}\left(x, \Sigma_{T}^{t, y, z}\right) \\
& \times \int_{t}^{T}\left\{2 d_{2}\left(\gamma_{1} f_{1}\right)\left(v_{\alpha}^{1, t, y}\right) \frac{\partial F_{[\alpha, T]}^{t, y, z}}{\partial y}+d_{1} \gamma_{1}\left(v_{\alpha}^{1, t, y}\right) f_{2}\left(v_{\alpha}^{2, t, z}\right) \frac{\partial \Sigma_{[\alpha, T]}^{t, y, z}}{\partial y}\right\} \\
& \left.\times\left[\frac{\partial v_{\alpha}^{1, t, y}}{\partial y}\right]^{-1} d \alpha\right] \\
& \theta_{13}(t, x, y, z)=-\mathbf{E}\left[\frac{\left(2 d_{1}+d_{2}-d_{1}^{2} d_{2}\right) \mathcal{N}^{\prime}\left(d_{2}\right)}{2\left(\Sigma_{T}^{t, y, z}\right)^{2}}\left(x, \Sigma_{T}^{t, y, z}\right)\right. \\
& \times \int_{t}^{T}\left\{2 f_{1}\left(v_{\alpha}^{1, t, y}\right) f_{2}\left(v_{\alpha}^{2, t, z}\right) c_{[\alpha, T]}^{2, t, z}\right. \\
& \left.+\left(\gamma_{2} f_{2}\right)\left(v_{\alpha}^{2, t, z}\right) F_{[\alpha, T]}^{t, y, z} \frac{\partial \Sigma_{[\alpha, T]}^{t, y, z}}{\partial z}\right\}\left[\frac{\partial v_{\alpha}^{2, t, z}}{\partial z}\right]^{-1} d \alpha \\
& -\frac{d_{1} \mathcal{N}^{\prime}\left(d_{2}\right)}{2 \Sigma_{T}^{t, y, z}}\left(x, \Sigma_{T}^{t, y, z}\right) \\
& \times \int_{t}^{T}\left\{2\left(\gamma_{2} f_{2}\right)\left(v_{\alpha}^{2, t, z}\right) \frac{\partial F_{[\alpha, T]}^{t, y, z}}{\partial z}+d_{2} \gamma_{2}\left(v_{\alpha}^{2, t, z}\right) f_{1}\left(v_{\alpha}^{1, t, y}\right) \frac{\partial \Sigma_{[\alpha, T]}^{t, y, z}}{\partial z}\right\}
\end{aligned}
$$




$$
\begin{aligned}
& \left.\times\left[\frac{\partial v_{\alpha}^{2, t, z}}{\partial z}\right]^{-1} d \alpha\right] \\
\theta_{23}(t, x, y, z)= & \mathbf{E}\left[\frac{\left(4 d_{1} d_{2}-3-d_{1}^{2} d_{2}^{2}+d_{1}^{2}+d_{2}^{2}\right) \mathcal{N}^{\prime}\left(d_{2}\right)}{2\left(\Sigma_{T}^{t, y, z}\right)^{\frac{5}{2}}}\left(x, \Sigma_{T}^{t, y, z}\right)\right. \\
& \times \int_{t}^{T}\left\{\left(\gamma_{1} f_{1}\right)\left(v_{\alpha}^{1, t, y}\right) c_{[\alpha, T]}^{2, t, z} \frac{\partial \Sigma_{[\alpha, T]}^{t, y, z}}{\partial y}\left(\gamma_{2} f_{2}\right)\left(v_{\alpha}^{2, t, z}\right) c_{[\alpha, T]}^{1, t, y} \frac{\partial \Sigma_{[\alpha, T]}^{t, y, z}}{\partial z}\right\} \\
& \left.\times\left[\frac{\partial v_{\alpha}^{1, t, y}}{\partial y} \frac{\partial v_{\alpha}^{2, t, z}}{\partial z}\right]^{-1} d \alpha\right]
\end{aligned}
$$

where for $\alpha \geq t$ and $w_{1}=y, w_{2}=z$, we denoted

$$
\begin{aligned}
F_{[\alpha, T]}^{t, y, z} & =\int_{\alpha}^{T} f_{1}\left(v_{\alpha_{1}}^{1, t, y}\right) f_{2}\left(v_{\alpha_{1}}^{2, t, z}\right) d \alpha_{1} \\
c_{[\alpha, T]}^{i, t, w_{i}} & \left.=\int_{\alpha}^{T} \int_{\alpha_{1}}^{T}\left(\gamma_{i} f_{i}\right)\left(v_{\alpha_{1}}^{i, t, w_{i}}\right)\left(f_{1} f_{1}^{\prime}\right)\left(v_{\alpha_{2}}^{i, t, w_{i}}\right) \frac{\partial v_{\alpha_{2}}^{i, t, w_{i}}}{\partial w_{i}}\right] d \alpha_{2} d \alpha_{1}, \\
\frac{\partial \Sigma_{[\alpha, T]}^{t, w_{1}, w_{2}}}{\partial w_{i}} & =2 \int_{\alpha}^{T}\left(f_{i} f_{i}^{\prime}\right)\left(v_{\alpha_{1}}^{i, t, w_{i}}\right) \frac{\partial v_{\alpha_{1}}^{i, t, w_{i}}}{\partial w_{i}} d \alpha_{1}
\end{aligned}
$$

\section{References}

Alos, E. (2006). A generalization of the Hull and White formula with applications to option pricing approximation. Finance and Stochastics, 10, 353-365.

Andersen, L. (2008). Simple and efficient simulation of the Heston stochastic volatility model. Journal of Computational Finance, 11(3), 1-42.

Antonelli, F., \& Scarlatti, S. (2009). Pricing option under stochastic volatility. A power series approach. Finance and Stochastics, 13(2), 269-303.

Bakshi, G. S., Cao, C., \& Chen, Z. (1997). Empirical performance of alternative option pricing models. Journal of Finance, 52, 2003-2049.

Bakshi, G. S., \& Madan, D. (2000). Spanning and derivative securities valuation. Journal of Financial Economics, 55, 205-238.

Broadie, M., \& Kaya, O. (2006). Exact simulation of stochastic volatility and other affine jump diffusion processes. Operations Research, 54(2), 217-231.

Chung, S. L., \& Wang, Y. W. (2008). Bounds and prices of currency cross-rate options. Journal of Banking \& Finance, 32, 631-642.

Duffie, D., Pan, J., \& Singleton, K. (2000). Transform analysis and asset pricing for affine jump-diffusions. Econometrica, 68(6), 1343-1376.

Evans, L. C. (1998). Partial differential equations. Providence, RI: Graduate Studies in Mathematics n.19 AMS.

Fisher, S. (1978). Call option pricing when the exercise price is uncertain, and the valuation of index bond. Journal of Finance, 33, 169-176.

Glasserman, P. (2004). Monte Carlo methods in financial engineering. New York: Springer. 
Heston S., L. A. (1993). Closed-form solution for options with stochastic volatility with applications to bond and currency options. Review in Financial Studies, 6, 327-343.

Heyde, C. C., \& Wong, B. (2004). On the martingale property of stochastic exponentials. Journal of Applied Probability, 41(3), 654-664.

Hull, J. C., \& White, A. (1987). The pricing of options on assets with stochastic volatilities. Journal of Finance, 42, 281-300.

Lord, R., Koekkoek, R. \& van Dijk, D.(2006). A comparison of biased simulation schemes for stochastic volatility models, working paper, Erasmus University Rotterdam, Rabobank International and Robeco Alternative Investments

Margrabe, W. (1978). The value of an option to exchange one asset for another. Journal of Finance, 33, 177186.

Maghsoodi, Y. (2007). Exact solutions of a martingzale stochastic volatility option problem and its empirical evaluation. Mathematical Finance, 17(2), 249-265.

Nualart, D. (1996). The Malliavin calculus and related topics. Probability and its applications. New York: Springer.

Poulsen, R. (2009). The Margrabe formula". Encyclopedia of Quantitative Finance (forthcoming). Available at "www.math.ku.dk/ rolf/EQF_Margrabe.pdf", preprint.

Stein, E., \& Stein, J. (1991). Stock price distributions with stochastic volatility: An analytic approach. Review of Financial Studies, 4, 727-752. 